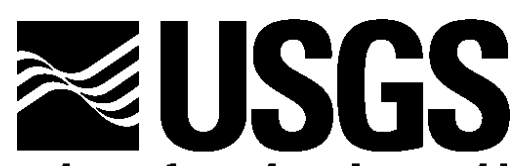

science for a changing world

\title{
Historical Ice-Out Dates for 29 Lakes in New England
}

Open-File Report 02-34

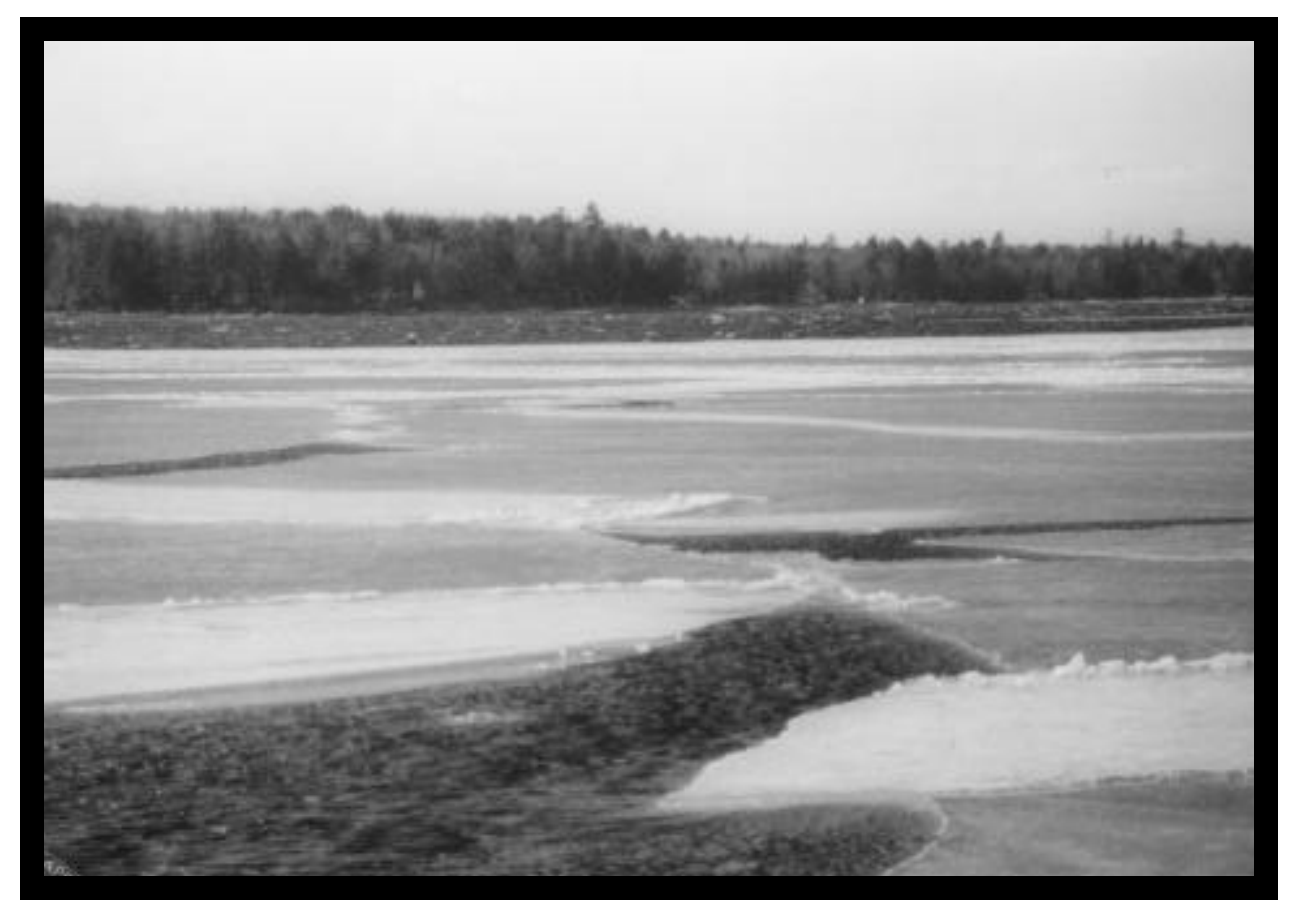

U.S. Department of the Interior U.S. Geological Survey 
Cover photograph: Ice-out on Sebago Lake, Jordan Bay, Spring 1985 By Glenn Hodgkins 
U.S. Department of the Interior

U.S. Geological Survey

\section{Historical Ice-Out Dates for 29 Lakes in New England}

By Glenn A. Hodgkins and Ivan C. James II

Open-File Report 02-34 


\section{U.S. DEPARTMENT OF THE INTERIOR \\ GALE A. NORTON, Secretary}

U.S. GEOLOGICAL SURVEY

Charles G. Groat, Director

The use of firm, trade, and brand names in this report is for identification purposes

only and does not constitute endorsement by the U.S. Government.

For additional information write to:

Copies of this report can be purchased from:

District Chief

U.S. Geological Survey

26 Ganneston Dr.

Augusta, ME 04330

http://me.water.usgs.gov
U.S. Geological Survey

Branch of Information Services

Box 25286, Federal Center

Denver, CO 80225 


\section{CONTENTS}

Abstract

\section{FIGURES}

1. Map showing location of lakes with long-term ice-out data in New England

\section{TABLES}

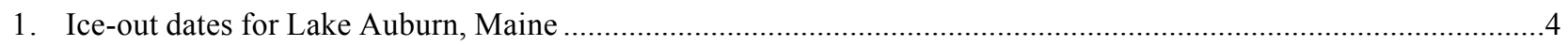

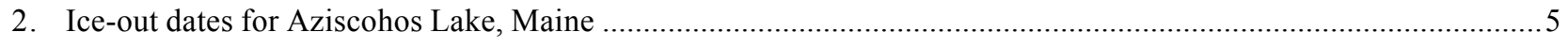

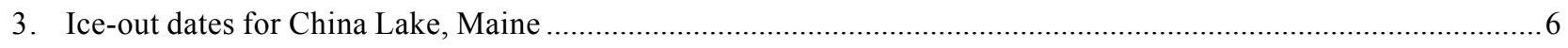

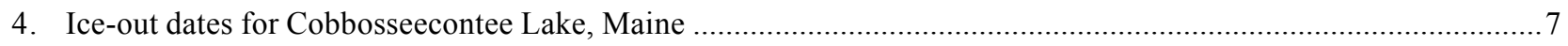

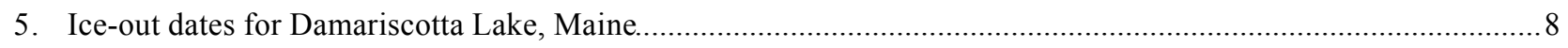

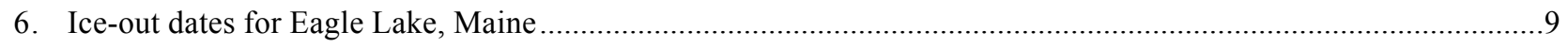

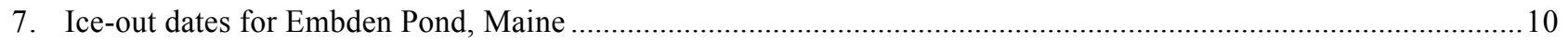

8. Ice-out dates for First Connecticut Lake, New Hampshire ....................................................................... 11

9. Ice-out dates for Houghtons (Hoosicwhisick) Pond, Massachusetts ............................................................12

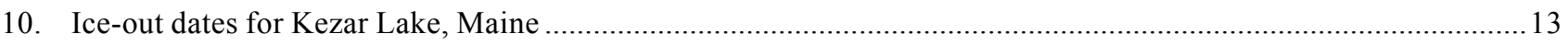

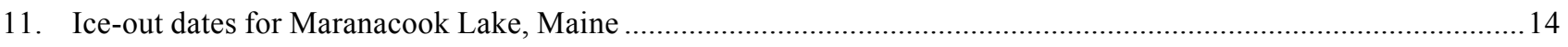

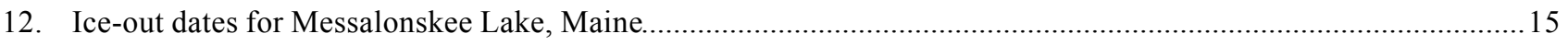

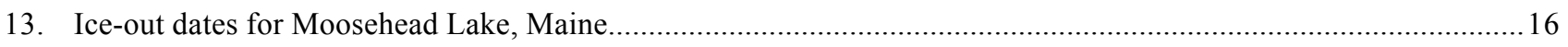

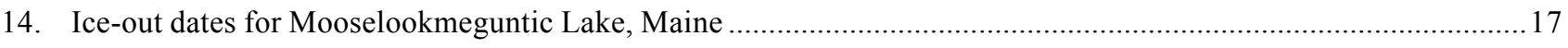

15. Ice-out dates for Pennesseewassee (Norway) Lake, Maine ............................................................................... 18

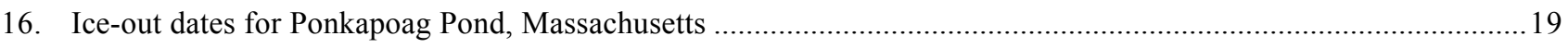

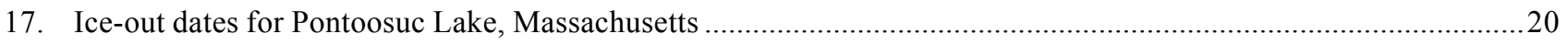

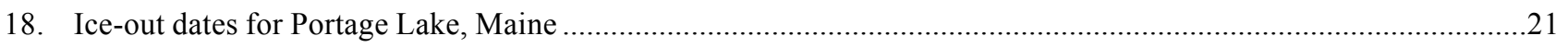

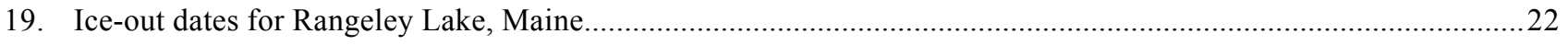

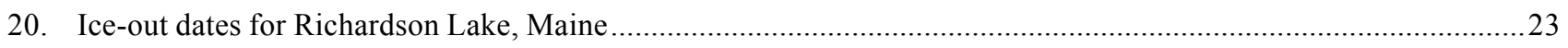

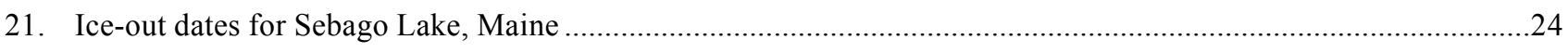

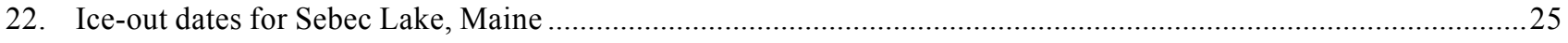

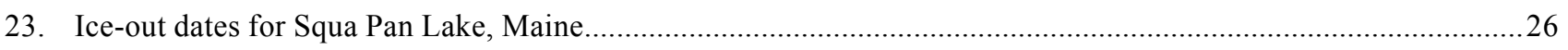

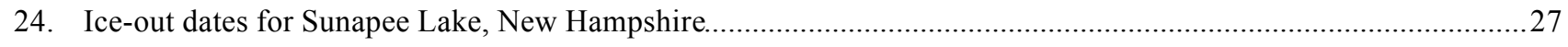

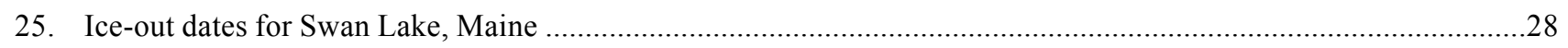

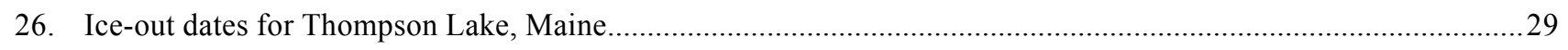

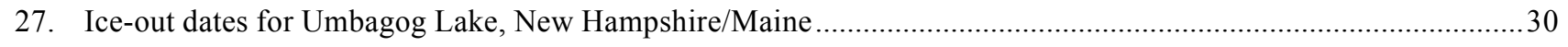

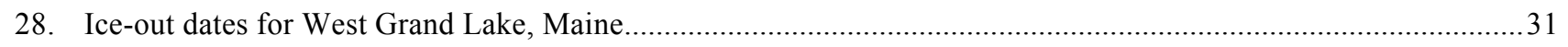

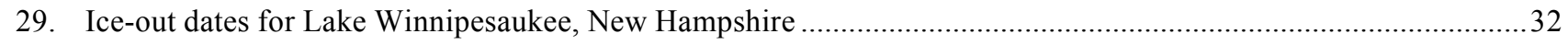





\title{
Historical Ice-Out Dates for 29 Lakes in New England
}

\author{
By Glenn A. Hodgkins and Ivan C. James II
}

\begin{abstract}
Historical ice-out dates for 29 lakes in New England were compiled and are presented in this report. The length of record for the lakes ranges from 64 to 163 years, with an average of 108 years. Many lakes in New England had their latest recorded ice-out date in 1888. Ice-out dates for lakes are an important hydrologic data series for climate researchers and other interested parties.
\end{abstract}

\section{INTRODUCTION}

Changes (or lack of changes) in long-term hydrologic data series in New England and other regions of the world are documented for several reasons, including validation of global circulation models at a regional scale and analysis of the effects of climatic changes on regional ecosystems. One longterm hydrologic data series that has not been well documented for the six New England states in published reports is lake ice-out dates. Lake ice-out dates are the annual dates in the spring when winter ice cover leaves a lake. Lake ice-out dates have been historically recorded in New England for practical reasons such as lake transportation and out of general interest.

Long-term data were compiled for 29 lakes in New England from individuals, water companies, newspapers, State fisheries agencies, power companies, and others. This report makes these data available to researchers and other interested parties. This report would not have been possible without the collection, compilation, and archiving of lake ice-out dates by many people over the past two centuries, especially Charles B. Fobes of Portland, Maine. Mr. Fobes' interest in lake ice-out dates extends back to the 1940's (Fobes, 1945). He recorded dates from observers at many lakes in Maine over the years and compiled older historical data at lakes. Much of the data presented in this report would have been lost or never collected without Mr. Fobes' work. Fifty-seven known individuals and organizations, and several unknown sources, contributed the data presented in this report. This study was funded by the U.S. Geological Survey Global Change Hydrology Program.

\section{COMPILATION OF LAKE ICE-OUT DATES}

Records from all six New England states (Connecticut, Maine, Massachusetts, New Hampshire, Rhode Island, and Vermont) were searched for longterm lake ice-out dates. This was done primarily by calling town officials, lake associations, newspapers, and fisheries agencies and following up on leads. It was not possible to search for records on all lakes. Particular attention was given to large lakes and lakes with nearby towns. Long-term lake ice-out dates were found for 29 lakes in New England (fig. 1). Most of these lakes are in Maine, with a few in New Hampshire and Massachusetts. No long-term records were found in Vermont, Connecticut, or Rhode Island. The ice-out records range from 64 to 163 years, with an average length of record of 108 years. Five lakes in New England have more than 150 years of data, and 16 have more than 100 years of data. The oldest record, for Sebago Lake, starts in 1807.

Ice-out definitions for individual lakes can vary over time and between observers. The exact definition (including the observation location) is not known with confidence in most cases. For lakes with overlapping 
data from more than one independent observer, the data that were judged to be most consistent over time with data from earlier and later time periods for that lake were used. No missing years of ice-out dates were estimated, and no adjustments to lake-ice observations were made, with the following exceptions: 19 years of data at Damariscotta Lake and 2 years at Cobbosseecontee Lake were filled in from a secondary observer and adjusted by the average difference in the dates between the primary and secondary observers; 1 year of data at China, Damariscotta, Portage, and Sebec Lakes and 2 years at Messalonskee Lake were not reasonable based on comparisons with other lakes in New England and were dropped from the dataset. Lake ice-out dates in Julian Days (January 1 of each calendar year is Julian Day 1, January 2 is Julian Day 2 and so forth) are given in tables 1 to 29. The latitude and longitude given in the tables are intended for general lake location only and do not necessarily represent the iceout observation location. The geographic location of the 29 lakes are shown in figure 1 . The numbers in figure 1 correspond to the table numbers for each lake.

\section{EXTREMES OF LAKE ICE-OUT DATES}

The differences between the earliest and latest ice-out dates for most lakes in New England were between 35 and 55 days. Seven lakes had their latest recorded ice-out date in 1888. Most remaining lakes with data for 1888 were within a few days of their record. Seven lakes in northern and mountainous areas of Maine and New Hampshire had their latest recorded ice-out dates in 1972. Nine lakes, primarily in southern Maine, had their earliest recorded ice-out dates in 1981, and eight lakes in northern and mountainous areas of Maine had their earliest recorded dates in 1945.

\section{REFERENCE CITED}

Fobes, C.B., 1945, The ice clearing dates of the Maine Lakes: Bulletin of the American Meteorological Society, v. 26, no. 8, p. 331-333. 


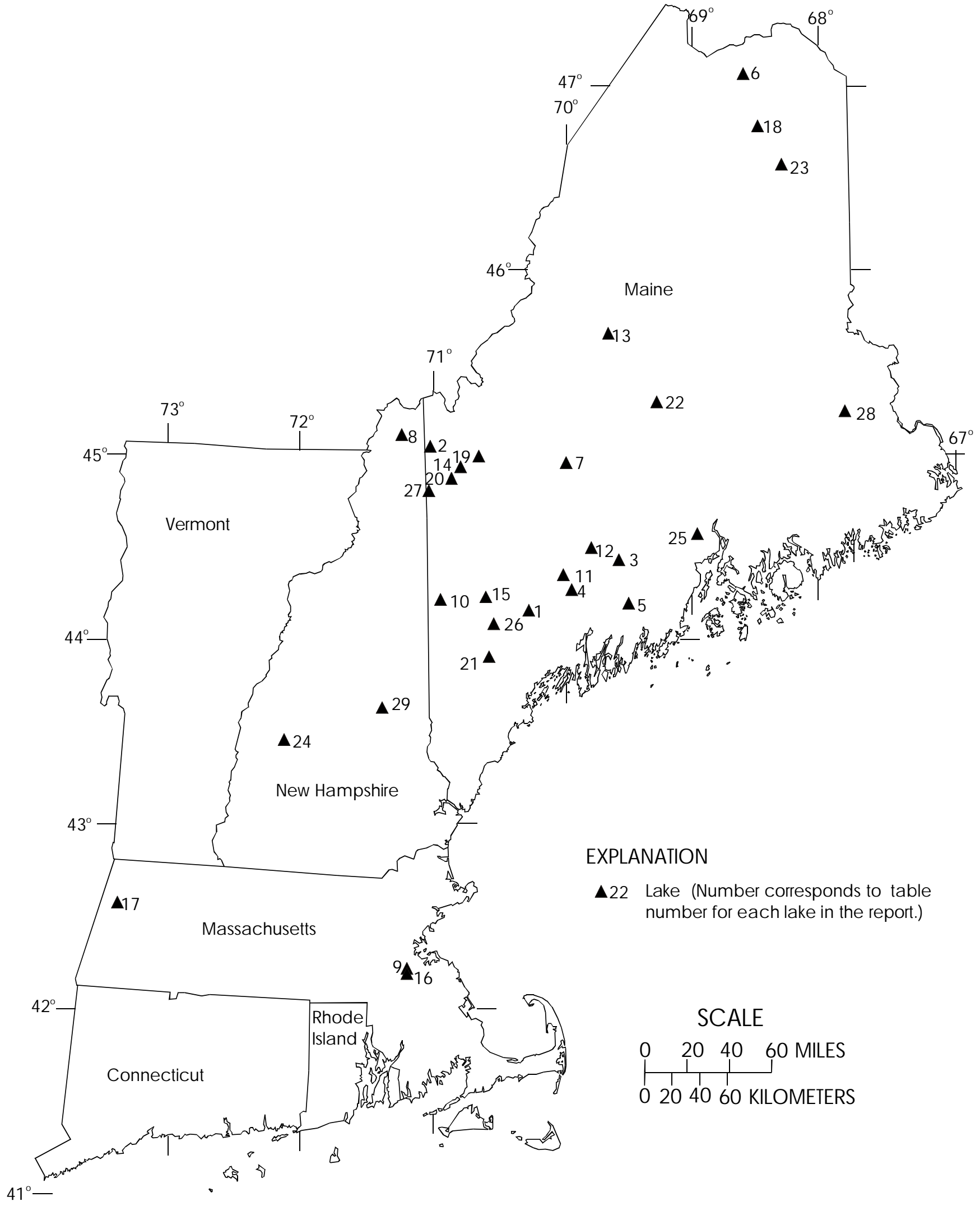

Figure 1. Location of lakes with long-term ice-out data in New England. 
Table 1. Ice-out dates for Lake Auburn, Maine

$[--$, no data]

Location of Lake.-Lat. $44^{\circ} 08^{\prime} 40^{\prime \prime}$, long. $70^{\circ} 15^{\prime} 00^{\prime \prime}$.

Period of Record.-1836 to 2000.

Years of Record.-158.

Observers.-Auburn Water District, Union Water Power Company, FPL Energy Maine, Lewiston Sun-Journal.

Earliest Ice-Out Date.-1981: 89.

Latest Ice-Out Date.-1874: 134.

\begin{tabular}{|c|c|c|c|c|c|c|c|c|c|}
\hline Year & $\begin{array}{c}\text { Julian } \\
\text { day }\end{array}$ & Year & $\begin{array}{c}\text { Julian } \\
\text { day }\end{array}$ & Year & $\begin{array}{c}\text { Julian } \\
\text { day }\end{array}$ & Year & $\begin{array}{c}\text { Julian } \\
\text { day }\end{array}$ & Year & $\begin{array}{c}\text { Julian } \\
\text { day }\end{array}$ \\
\hline 1807 & -- & 1846 & 105 & 1885 & 118 & 1924 & 112 & 1963 & 110 \\
\hline 1808 & -- & 1847 & 130 & 1886 & 114 & 1925 & 99 & 1964 & 117 \\
\hline 1809 & -- & 1848 & 108 & 1887 & 126 & 1926 & 122 & 1965 & 112 \\
\hline 1810 & -- & 1849 & 104 & 1888 & 132 & 1927 & 106 & 1966 & 110 \\
\hline 1811 & -- & 1850 & 121 & 1889 & 107 & 1928 & 111 & 1967 & 119 \\
\hline 1812 & -- & 1851 & 111 & 1890 & 116 & 1929 & 113 & 1968 & 105 \\
\hline 1813 & -- & 1852 & 128 & 1891 & 117 & 1930 & 104 & 1969 & 114 \\
\hline 1814 & -- & 1853 & 112 & 1892 & 112 & 1931 & 101 & 1970 & 117 \\
\hline 1815 & -- & 1854 & 126 & 1893 & 125 & 1932 & 111 & 1971 & 121 \\
\hline 1816 & -- & 1855 & 125 & 1894 & 114 & 1933 & 112 & 1972 & 127 \\
\hline 1817 & -- & 1856 & 116 & 1895 & 113 & 1934 & 113 & 1973 & 107 \\
\hline 1818 & -- & 1857 & 111 & 1896 & 116 & 1935 & 115 & 1974 & 100 \\
\hline 1819 & -- & 1858 & -- & 1897 & 116 & 1936 & 105 & 1975 & 119 \\
\hline 1820 & -- & 1859 & 120 & 1898 & 108 & 1937 & 117 & 1976 & 108 \\
\hline 1821 & -- & 1860 & 115 & 1899 & 120 & 1938 & 109 & 1977 & 109 \\
\hline 1822 & -- & 1861 & 115 & 1900 & 116 & 1939 & 126 & 1978 & 118 \\
\hline 1823 & -- & 1862 & 126 & 1901 & 105 & 1940 & 127 & 1979 & 113 \\
\hline 1824 & -- & 1863 & 123 & 1902 & 94 & 1941 & 104 & 1980 & 104 \\
\hline 1825 & -- & 1864 & -- & 1903 & 94 & 1942 & 108 & 1981 & 89 \\
\hline 1826 & -- & 1865 & -- & 1904 & 122 & 1943 & 117 & 1982 & 118 \\
\hline 1827 & -- & 1866 & -- & 1905 & 113 & 1944 & 120 & 1983 & 97 \\
\hline 1828 & -- & 1867 & -- & 1906 & 114 & 1945 & 93 & 1984 & 111 \\
\hline 1829 & -- & 1868 & -- & 1907 & 120 & 1946 & 101 & 1985 & 94 \\
\hline 1830 & -- & 1869 & -- & 1908 & 115 & 1947 & 108 & 1986 & 104 \\
\hline 1831 & -- & 1870 & 114 & 1909 & 110 & 1948 & 102 & 1987 & 102 \\
\hline 1832 & -- & 1871 & 106 & 1910 & 95 & 1949 & 100 & 1988 & 103 \\
\hline 1833 & -- & 1872 & 122 & 1911 & 119 & 1950 & 114 & 1989 & 112 \\
\hline 1834 & -- & 1873 & 121 & 1912 & 114 & 1951 & 99 & 1990 & 104 \\
\hline 1835 & -- & 1874 & 134 & 1913 & 108 & 1952 & 111 & 1991 & 98 \\
\hline 1836 & 129 & 1875 & 126 & 1914 & 114 & 1953 & 91 & 1992 & 101 \\
\hline 1837 & 120 & 1876 & 126 & 1915 & 103 & 1954 & 109 & 1993 & 111 \\
\hline 1838 & 117 & 1877 & 110 & 1916 & 113 & 1955 & 107 & 1994 & 113 \\
\hline 1839 & 108 & 1878 & 105 & 1917 & 117 & 1956 & 120 & 1995 & 104 \\
\hline 1840 & 119 & 1879 & 128 & 1918 & 114 & 1957 & 108 & 1996 & 100 \\
\hline 1841 & 120 & 1880 & 112 & 1919 & 101 & 1958 & 107 & 1997 & 114 \\
\hline 1842 & 109 & 1881 & 113 & 1920 & 118 & 1959 & 114 & 1998 & 98 \\
\hline 1843 & 126 & 1882 & 122 & 1921 & 93 & 1960 & 122 & 1999 & 97 \\
\hline 1844 & 111 & 1883 & 121 & 1922 & 107 & 1961 & 121 & 2000 & 97 \\
\hline 1845 & 122 & 1884 & 119 & 1923 & 118 & 1962 & 113 & & \\
\hline
\end{tabular}


Table 2. Ice-out dates for Aziscohos Lake, Maine

[--, no data]

Location of Lake.-Lat. $45^{\circ} 01^{\prime} 16^{\prime \prime}$, long. $71^{\circ} 00^{\prime} 51^{\prime \prime}$.

Period of Record.-1913 to 2000.

Years of Record.-86.

Observers.-Union Water Power Company, FPL Energy Maine.

Earliest Ice-Out Date.-1945:104.

Latest Ice-Out Date.-1972:141.

\begin{tabular}{|c|c|c|c|c|c|c|c|c|c|}
\hline Year & $\begin{array}{c}\text { Julian } \\
\text { day }\end{array}$ & Year & $\begin{array}{c}\text { Julian } \\
\text { day }\end{array}$ & Year & $\begin{array}{c}\text { Julian } \\
\text { day }\end{array}$ & Year & $\begin{array}{c}\begin{array}{c}\text { Julian } \\
\text { day }\end{array} \\
\end{array}$ & Year & $\begin{array}{c}\begin{array}{c}\text { Julian } \\
\text { day }\end{array} \\
\end{array}$ \\
\hline 1807 & -- & 1846 & -- & 1885 & -- & 1924 & 130 & 1963 & 127 \\
\hline 1808 & -- & 1847 & -- & 1886 & -- & 1925 & 118 & 1964 & 128 \\
\hline 1809 & -- & 1848 & -- & 1887 & -- & 1926 & 136 & 1965 & 126 \\
\hline 1810 & -- & 1849 & -- & 1888 & -- & 1927 & 112 & 1966 & 137 \\
\hline 1811 & -- & 1850 & -- & 1889 & -- & 1928 & 135 & 1967 & 127 \\
\hline 1812 & -- & 1851 & -- & 1890 & -- & 1929 & 131 & 1968 & 114 \\
\hline 1813 & -- & 1852 & -- & 1891 & -- & 1930 & 125 & 1969 & 131 \\
\hline 1814 & -- & 1853 & -- & 1892 & -- & 1931 & 113 & 1970 & 136 \\
\hline 1815 & -- & 1854 & -- & 1893 & -- & 1932 & 129 & 1971 & 134 \\
\hline 1816 & -- & 1855 & -- & 1894 & -- & 1933 & 131 & 1972 & 141 \\
\hline 1817 & -- & 1856 & -- & 1895 & -- & 1934 & -- & 1973 & 123 \\
\hline 1818 & -- & 1857 & -- & 1896 & -- & 1935 & -- & 1974 & 135 \\
\hline 1819 & -- & 1858 & -- & 1897 & -- & 1936 & 122 & 1975 & 133 \\
\hline 1820 & -- & 1859 & -- & 1898 & -- & 1937 & 133 & 1976 & 118 \\
\hline 1821 & -- & 1860 & -- & 1899 & -- & 1938 & 120 & 1977 & 126 \\
\hline 1822 & -- & 1861 & -- & 1900 & -- & 1939 & 133 & 1978 & 136 \\
\hline 1823 & -- & 1862 & -- & 1901 & -- & 1940 & 136 & 1979 & 122 \\
\hline 1824 & -- & 1863 & -- & 1902 & -- & 1941 & 118 & 1980 & 125 \\
\hline 1825 & -- & 1864 & -- & 1903 & -- & 1942 & 125 & 1981 & 121 \\
\hline 1826 & -- & 1865 & -- & 1904 & -- & 1943 & 138 & 1982 & 130 \\
\hline 1827 & -- & 1866 & -- & 1905 & -- & 1944 & 132 & 1983 & 126 \\
\hline 1828 & -- & 1867 & -- & 1906 & -- & 1945 & 104 & 1984 & 123 \\
\hline 1829 & -- & 1868 & -- & 1907 & -- & 1946 & 122 & 1985 & 117 \\
\hline 1830 & -- & 1869 & -- & 1908 & -- & 1947 & 137 & 1986 & 116 \\
\hline 1831 & -- & 1870 & -- & 1909 & -- & 1948 & 116 & 1987 & 110 \\
\hline 1832 & -- & 1871 & -- & 1910 & -- & 1949 & 118 & 1988 & 120 \\
\hline 1833 & -- & 1872 & -- & 1911 & -- & 1950 & 128 & 1989 & 128 \\
\hline 1834 & -- & 1873 & -- & 1912 & -- & 1951 & 122 & 1990 & 124 \\
\hline 1835 & -- & 1874 & -- & 1913 & 122 & 1952 & 121 & 1991 & 123 \\
\hline 1836 & -- & 1875 & -- & 1914 & 128 & 1953 & 114 & 1992 & 135 \\
\hline 1837 & -- & 1876 & -- & 1915 & 116 & 1954 & 128 & 1993 & 123 \\
\hline 1838 & -- & 1877 & -- & 1916 & 129 & 1955 & 122 & 1994 & 131 \\
\hline 1839 & -- & 1878 & -- & 1917 & 134 & 1956 & 134 & 1995 & 122 \\
\hline 1840 & -- & 1879 & -- & 1918 & 119 & 1957 & 121 & 1996 & 131 \\
\hline 1841 & -- & 1880 & -- & 1919 & 125 & 1958 & 127 & 1997 & 133 \\
\hline 1842 & -- & 1881 & -- & 1920 & 131 & 1959 & 126 & 1998 & 113 \\
\hline 1843 & -- & 1882 & -- & 1921 & 106 & 1960 & 123 & 1999 & 122 \\
\hline 1844 & -- & 1883 & -- & 1922 & 123 & 1961 & 136 & 2000 & 122 \\
\hline 1845 & -- & 1884 & -- & 1923 & 126 & 1962 & 129 & & \\
\hline
\end{tabular}


Table 3. Ice-out dates for China Lake, Maine

$[--$, no data]

Location of Lake.-Lat. $44^{\circ} 26^{\prime} 02^{\prime \prime}$, long. $69^{\circ} 32^{\prime} 44^{\prime \prime}$.

Period of Record.-1874 to 2000.

Years of Record.-74.

Observers. - The Town Line, Bill Foster, Captain James Allen, Theresa Plaisted.

Earliest Ice-Out Date.-1981: 77.

Latest Ice-Out Date.-1875: 126.

\begin{tabular}{|c|c|c|c|c|c|c|c|c|c|}
\hline Year & $\begin{array}{c}\text { Julian } \\
\text { day }\end{array}$ & Year & $\begin{array}{c}\text { Julian } \\
\text { day }\end{array}$ & Year & $\begin{array}{c}\text { Julian } \\
\text { day }\end{array}$ & Year & $\begin{array}{c}\text { Julian } \\
\text { day }\end{array}$ & Year & $\begin{array}{c}\text { Julian } \\
\text { day }\end{array}$ \\
\hline 1807 & -- & 1846 & -- & 1885 & -- & 1924 & -- & 1963 & 112 \\
\hline 1808 & -- & 1847 & -- & 1886 & -- & 1925 & -- & 1964 & 112 \\
\hline 1809 & -- & 1848 & -- & 1887 & -- & 1926 & -- & 1965 & 108 \\
\hline 1810 & -- & 1849 & -- & 1888 & -- & 1927 & -- & 1966 & 108 \\
\hline 1811 & -- & 1850 & -- & 1889 & -- & 1928 & -- & 1967 & 119 \\
\hline 1812 & -- & 1851 & -- & 1890 & -- & 1929 & -- & 1968 & 104 \\
\hline 1813 & -- & 1852 & -- & 1891 & -- & 1930 & -- & 1969 & 113 \\
\hline 1814 & -- & 1853 & -- & 1892 & -- & 1931 & -- & 1970 & 113 \\
\hline 1815 & -- & 1854 & -- & 1893 & -- & 1932 & 118 & 1971 & 120 \\
\hline 1816 & -- & 1855 & -- & 1894 & -- & 1933 & 110 & 1972 & 122 \\
\hline 1817 & -- & 1856 & -- & 1895 & -- & 1934 & 119 & 1973 & 98 \\
\hline 1818 & -- & 1857 & -- & 1896 & -- & 1935 & 115 & 1974 & 92 \\
\hline 1819 & -- & 1858 & -- & 1897 & -- & 1936 & 95 & 1975 & 113 \\
\hline 1820 & -- & 1859 & -- & 1898 & -- & 1937 & 110 & 1976 & 102 \\
\hline 1821 & -- & 1860 & -- & 1899 & -- & 1938 & 110 & 1977 & 108 \\
\hline 1822 & -- & 1861 & -- & 1900 & -- & 1939 & 124 & 1978 & 111 \\
\hline 1823 & -- & 1862 & -- & 1901 & -- & 1940 & -- & 1979 & 102 \\
\hline 1824 & -- & 1863 & -- & 1902 & -- & 1941 & 106 & 1980 & 101 \\
\hline 1825 & -- & 1864 & -- & 1903 & -- & 1942 & -- & 1981 & 77 \\
\hline 1826 & -- & 1865 & -- & 1904 & -- & 1943 & -- & 1982 & 112 \\
\hline 1827 & -- & 1866 & -- & 1905 & -- & 1944 & -- & 1983 & 91 \\
\hline 1828 & -- & 1867 & -- & 1906 & -- & 1945 & 92 & 1984 & 108 \\
\hline 1829 & -- & 1868 & -- & 1907 & -- & 1946 & -- & 1985 & 96 \\
\hline 1830 & -- & 1869 & -- & 1908 & -- & 1947 & 102 & 1986 & 98 \\
\hline 1831 & -- & 1870 & -- & 1909 & -- & 1948 & 99 & 1987 & 96 \\
\hline 1832 & -- & 1871 & -- & 1910 & -- & 1949 & 96 & 1988 & 97 \\
\hline 1833 & -- & 1872 & -- & 1911 & -- & 1950 & 104 & 1989 & 112 \\
\hline 1834 & -- & 1873 & -- & 1912 & -- & 1951 & 99 & 1990 & 101 \\
\hline 1835 & -- & 1874 & 112 & 1913 & -- & 1952 & 110 & 1991 & 98 \\
\hline 1836 & -- & 1875 & 126 & 1914 & -- & 1953 & 78 & 1992 & 106 \\
\hline 1837 & -- & 1876 & 121 & 1915 & -- & 1954 & 109 & 1993 & 111 \\
\hline 1838 & -- & 1877 & 106 & 1916 & -- & 1955 & 103 & 1994 & 110 \\
\hline 1839 & -- & 1878 & 102 & 1917 & -- & 1956 & 118 & 1995 & 99 \\
\hline 1840 & -- & 1879 & 123 & 1918 & -- & 1957 & 100 & 1996 & 96 \\
\hline 1841 & -- & 1880 & 112 & 1919 & -- & 1958 & 106 & 1997 & 113 \\
\hline 1842 & -- & 1881 & 109 & 1920 & -- & 1959 & 112 & 1998 & 99 \\
\hline 1843 & -- & 1882 & -- & 1921 & 87 & 1960 & 112 & 1999 & 92 \\
\hline 1844 & -- & 1883 & 119 & 1922 & -- & 1961 & 120 & 2000 & 95 \\
\hline 1845 & -- & 1884 & -- & 1923 & -- & 1962 & 110 & & \\
\hline
\end{tabular}


Table 4. Ice-out dates for Cobbosseecontee Lake, Maine

$[--$, no data $]$

Location of Lake.-Lat. $44^{\circ} 16^{\prime} 18^{\prime \prime}$, long. $69^{\circ} 55^{\prime} 46^{\prime \prime}$.

Period of Record.- -1840 to 2000.

Years of Record.-159.

Observers.-Sara Farr, Mary Oatway, Bill Schenck.

Earliest Ice-Out Date.-1981: 75.

Latest Ice-Out Date.-1888: 130.

Comments. -1974 and 1994 were filled in from a secondary observer and adjusted by the average difference in the dates between the primary and secondary observers.

\begin{tabular}{|c|c|c|c|c|c|c|c|c|c|}
\hline Year & $\begin{array}{c}\text { Julian } \\
\text { day }\end{array}$ & Year & $\begin{array}{c}\text { Julian } \\
\text { day }\end{array}$ & Year & $\begin{array}{c}\text { Julian } \\
\text { day }\end{array}$ & Year & $\begin{array}{c}\text { Julian } \\
\text { day }\end{array}$ & Year & $\begin{array}{c}\text { Julian } \\
\text { day }\end{array}$ \\
\hline 1807 & -- & 1846 & 107 & 1885 & 119 & 1924 & 110 & 1963 & 111 \\
\hline 1808 & -- & 1847 & 128 & 1886 & 110 & 1925 & 96 & 1964 & 112 \\
\hline 1809 & -- & 1848 & -- & 1887 & 127 & 1926 & 123 & 1965 & 109 \\
\hline 1810 & -- & 1849 & -- & 1888 & 130 & 1927 & 106 & 1966 & 109 \\
\hline 1811 & -- & 1850 & 121 & 1889 & 105 & 1928 & 111 & 1967 & 119 \\
\hline 1812 & -- & 1851 & 111 & 1890 & 116 & 1929 & 111 & 1968 & 104 \\
\hline 1813 & -- & 1852 & 124 & 1891 & 118 & 1930 & 103 & 1969 & 114 \\
\hline 1814 & -- & 1853 & 110 & 1892 & 109 & 1931 & 102 & 1970 & 120 \\
\hline 1815 & -- & 1854 & 128 & 1893 & 124 & 1932 & 111 & 1971 & 120 \\
\hline 1816 & -- & 1855 & 115 & 1894 & 114 & 1933 & 109 & 1972 & 120 \\
\hline 1817 & -- & 1856 & 117 & 1895 & 111 & 1934 & 112 & 1973 & 107 \\
\hline 1818 & -- & 1857 & 107 & 1896 & 113 & 1935 & 115 & 1974 & 96 \\
\hline 1819 & -- & 1858 & 108 & 1897 & 114 & 1936 & 103 & 1975 & 115 \\
\hline 1820 & -- & 1859 & 120 & 1898 & 106 & 1937 & 113 & 1976 & 107 \\
\hline 1821 & -- & 1860 & 114 & 1899 & 119 & 1938 & 108 & 1977 & 111 \\
\hline 1822 & -- & 1861 & 115 & 1900 & 114 & 1939 & 124 & 1978 & 117 \\
\hline 1823 & -- & 1862 & 126 & 1901 & 110 & 1940 & 123 & 1979 & 107 \\
\hline 1824 & -- & 1863 & 122 & 1902 & 92 & 1941 & 102 & 1980 & 100 \\
\hline 1825 & -- & 1864 & 114 & 1903 & 95 & 1942 & 103 & 1981 & 75 \\
\hline 1826 & -- & 1865 & 103 & 1904 & 119 & 1943 & 116 & 1982 & 112 \\
\hline 1827 & -- & 1866 & 108 & 1905 & 111 & 1944 & 119 & 1983 & 100 \\
\hline 1828 & -- & 1867 & 117 & 1906 & 114 & 1945 & 93 & 1984 & 107 \\
\hline 1829 & -- & 1868 & 122 & 1907 & 121 & 1946 & 97 & 1985 & 95 \\
\hline 1830 & -- & 1869 & 119 & 1908 & 112 & 1947 & 104 & 1986 & 101 \\
\hline 1831 & -- & 1870 & 114 & 1909 & 109 & 1948 & 100 & 1987 & 101 \\
\hline 1832 & -- & 1871 & 99 & 1910 & 94 & 1949 & 101 & 1988 & 102 \\
\hline 1833 & -- & 1872 & 124 & 1911 & 118 & 1950 & 111 & 1989 & 113 \\
\hline 1834 & -- & 1873 & 122 & 1912 & 115 & 1951 & 96 & 1990 & 104 \\
\hline 1835 & -- & 1874 & 124 & 1913 & 107 & 1952 & 111 & 1991 & 97 \\
\hline 1836 & -- & 1875 & 122 & 1914 & 116 & 1953 & 87 & 1992 & 100 \\
\hline 1837 & -- & 1876 & 122 & 1915 & 104 & 1954 & 109 & 1993 & 111 \\
\hline 1838 & -- & 1877 & 108 & 1916 & 113 & 1955 & 106 & 1994 & 114 \\
\hline 1839 & -- & 1878 & 104 & 1917 & 116 & 1956 & 119 & 1995 & 99 \\
\hline 1840 & 106 & 1879 & 125 & 1918 & 118 & 1957 & 103 & 1996 & 98 \\
\hline 1841 & 118 & 1880 & 113 & 1919 & 100 & 1958 & 106 & 1997 & 113 \\
\hline 1842 & 106 & 1881 & 111 & 1920 & 119 & 1959 & 112 & 1998 & 95 \\
\hline 1843 & 125 & 1882 & 118 & 1921 & 91 & 1960 & 115 & 1999 & 94 \\
\hline 1844 & 113 & 1883 & 121 & 1922 & 105 & 1961 & 120 & 2000 & 94 \\
\hline 1845 & 122 & 1884 & 117 & 1923 & 116 & 1962 & 112 & & \\
\hline
\end{tabular}


Table 5. Ice-out dates for Damariscotta Lake, Maine

[--, no data]

Location of Lake.-Lat. $44^{\circ} 10^{\prime} 57^{\prime \prime}$, long. $69^{\circ} 28^{\prime} 45^{\prime \prime}$.

Period of Record.-1837 to 2000.

Years of Record.-163.

Observers and Ice-Out Definition. - Fred Jackson and family (three generations), James Birkett. Ice-out for most years thought to be observed from the northern end of the lake.

Earliest Ice-Out Date.-1981: 72.

Latest Ice-Out Date. - 1888: 126.

Comments. - 1959, 1962-68, 1970-78, and 1997-98 were filled in from a secondary observer and adjusted by the average difference in the dates between the primary and secondary observers.

\begin{tabular}{|c|c|c|c|c|c|c|c|c|c|}
\hline Year & $\begin{array}{c}\text { Julian } \\
\text { day }\end{array}$ & Year & $\begin{array}{c}\text { Julian } \\
\text { day }\end{array}$ & Year & $\begin{array}{c}\text { Julian } \\
\text { day }\end{array}$ & Year & $\begin{array}{c}\text { Julian } \\
\text { day }\end{array}$ & Year & $\begin{array}{c}\text { Julian } \\
\text { day }\end{array}$ \\
\hline 1807 & -- & 1846 & 100 & 1885 & 115 & 1924 & 109 & 1963 & 114 \\
\hline 1808 & -- & 1847 & 123 & 1886 & 114 & 1925 & 93 & 1964 & 112 \\
\hline 1809 & -- & 1848 & 102 & 1887 & 124 & 1926 & 122 & 1965 & 103 \\
\hline 1810 & -- & 1849 & 98 & 1888 & 126 & 1927 & -- & 1966 & 110 \\
\hline 1811 & -- & 1850 & 118 & 1889 & 102 & 1928 & 103 & 1967 & 121 \\
\hline 1812 & -- & 1851 & 106 & 1890 & 112 & 1929 & 106 & 1968 & 104 \\
\hline 1813 & -- & 1852 & 118 & 1891 & 108 & 1930 & 100 & 1969 & 110 \\
\hline 1814 & -- & 1853 & 103 & 1892 & 102 & 1931 & 101 & 1970 & 112 \\
\hline 1815 & -- & 1854 & 124 & 1893 & 119 & 1932 & 103 & 1971 & 118 \\
\hline 1816 & -- & 1855 & 105 & 1894 & 110 & 1933 & 97 & 1972 & 115 \\
\hline 1817 & -- & 1856 & 117 & 1895 & 111 & 1934 & 107 & 1973 & 96 \\
\hline 1818 & -- & 1857 & 104 & 1896 & 110 & 1935 & 112 & 1974 & 77 \\
\hline 1819 & -- & 1858 & 119 & 1897 & 109 & 1936 & 92 & 1975 & 111 \\
\hline 1820 & -- & 1859 & 100 & 1898 & 102 & 1937 & 108 & 1976 & 99 \\
\hline 1821 & -- & 1860 & 116 & 1899 & 115 & 1938 & 100 & 1977 & 108 \\
\hline 1822 & -- & 1861 & 108 & 1900 & 102 & 1939 & 121 & 1978 & 116 \\
\hline 1823 & -- & 1862 & 122 & 1901 & 108 & 1940 & 118 & 1979 & 97 \\
\hline 1824 & -- & 1863 & 114 & 1902 & 86 & 1941 & 103 & 1980 & 97 \\
\hline 1825 & -- & 1864 & 110 & 1903 & 85 & 1942 & 100 & 1981 & 72 \\
\hline 1826 & -- & 1865 & 97 & 1904 & 117 & 1943 & 112 & 1982 & 112 \\
\hline 1827 & -- & 1866 & 106 & 1905 & 111 & 1944 & 114 & 1983 & 85 \\
\hline 1828 & -- & 1867 & 113 & 1906 & 111 & 1945 & 91 & 1984 & 103 \\
\hline 1829 & -- & 1868 & 122 & 1907 & 120 & 1946 & 90 & 1985 & 93 \\
\hline 1830 & -- & 1869 & 115 & 1908 & 107 & 1947 & 101 & 1986 & 96 \\
\hline 1831 & -- & 1870 & 108 & 1909 & 104 & 1948 & 95 & 1987 & 101 \\
\hline 1832 & -- & 1871 & 86 & 1910 & 92 & 1949 & 96 & 1988 & 97 \\
\hline 1833 & -- & 1872 & 124 & 1911 & 118 & 1950 & 108 & 1989 & 106 \\
\hline 1834 & -- & 1873 & 119 & 1912 & 110 & 1951 & 92 & 1990 & 100 \\
\hline 1835 & -- & 1874 & 116 & 1913 & 95 & 1952 & 108 & 1991 & 96 \\
\hline 1836 & -- & 1875 & 122 & 1914 & 111 & 1953 & 84 & 1992 & 100 \\
\hline 1837 & 115 & 1876 & 118 & 1915 & 91 & 1954 & 99 & 1993 & 111 \\
\hline 1838 & 110 & 1877 & 105 & 1916 & 109 & 1955 & 101 & 1994 & 106 \\
\hline 1839 & 105 & 1878 & 97 & 1917 & 114 & 1956 & 117 & 1995 & 95 \\
\hline 1840 & 103 & 1879 & 120 & 1918 & 114 & 1957 & 96 & 1996 & 98 \\
\hline 1841 & 115 & 1880 & 108 & 1919 & 95 & 1958 & 99 & 1997 & 111 \\
\hline 1842 & 107 & 1881 & 106 & 1920 & 114 & 1959 & 109 & 1998 & 73 \\
\hline 1843 & 121 & 1882 & 115 & 1921 & 87 & 1960 & 111 & 1999 & 81 \\
\hline 1844 & 105 & 1883 & 118 & 1922 & 102 & 1961 & 119 & 2000 & 94 \\
\hline 1845 & 115 & 1884 & 115 & 1923 & 115 & 1962 & 104 & & \\
\hline
\end{tabular}


Table 6. Ice-out dates for Eagle Lake, Maine

[--, no data]

Location of Lake.-Lat. $47^{\circ} 02^{\prime} 54^{\prime \prime}$, long. $68^{\circ} 35^{\prime} 07^{\prime \prime}$.

Period of Record.-1922 to 2000.

Years of Record.-67.

Observers.-Maine Department of Inland Fisheries and Wildlife, Derwood Humphrey, S. C. Michaud, John M. Caron.

Earliest Ice-Out Date.-1945: 104.

Latest Ice-Out Date._-1974: 143.

\begin{tabular}{|c|c|c|c|c|c|c|c|c|c|}
\hline Year & $\begin{array}{c}\text { Julian } \\
\text { day }\end{array}$ & Year & $\begin{array}{c}\text { Julian } \\
\text { day }\end{array}$ & Year & $\begin{array}{c}\text { Julian } \\
\text { day }\end{array}$ & Year & $\begin{array}{c}\text { Julian } \\
\text { day }\end{array}$ & Year & $\begin{array}{c}\text { Julian } \\
\text { day }\end{array}$ \\
\hline 1807 & -- & 1846 & -- & 1885 & -- & 1924 & 134 & 1963 & -- \\
\hline 1808 & -- & 1847 & -- & 1886 & -- & 1925 & 124 & 1964 & -- \\
\hline 1809 & -- & 1848 & -- & 1887 & -- & 1926 & 138 & 1965 & -- \\
\hline 1810 & -- & 1849 & -- & 1888 & -- & 1927 & 122 & 1966 & -- \\
\hline 1811 & -- & 1850 & -- & 1889 & -- & 1928 & 129 & 1967 & -- \\
\hline 1812 & -- & 1851 & -- & 1890 & -- & 1929 & 127 & 1968 & -- \\
\hline 1813 & -- & 1852 & -- & 1891 & -- & 1930 & 127 & 1969 & 134 \\
\hline 1814 & -- & 1853 & -- & 1892 & -- & 1931 & 114 & 1970 & 130 \\
\hline 1815 & -- & 1854 & -- & 1893 & -- & 1932 & 127 & 1971 & 138 \\
\hline 1816 & -- & 1855 & -- & 1894 & -- & 1933 & 128 & 1972 & 141 \\
\hline 1817 & -- & 1856 & -- & 1895 & -- & 1934 & 122 & 1973 & 129 \\
\hline 1818 & -- & 1857 & -- & 1896 & -- & 1935 & 126 & 1974 & 143 \\
\hline 1819 & -- & 1858 & -- & 1897 & -- & 1936 & 123 & 1975 & 135 \\
\hline 1820 & -- & 1859 & -- & 1898 & -- & 1937 & 127 & 1976 & 124 \\
\hline 1821 & -- & 1860 & -- & 1899 & -- & 1938 & 119 & 1977 & 131 \\
\hline 1822 & -- & 1861 & -- & 1900 & -- & 1939 & 132 & 1978 & 134 \\
\hline 1823 & -- & 1862 & -- & 1901 & -- & 1940 & 134 & 1979 & 124 \\
\hline 1824 & -- & 1863 & -- & 1902 & -- & 1941 & 128 & 1980 & 128 \\
\hline 1825 & -- & 1864 & -- & 1903 & -- & 1942 & 124 & 1981 & 122 \\
\hline 1826 & -- & 1865 & -- & 1904 & -- & 1943 & 138 & 1982 & 134 \\
\hline 1827 & -- & 1866 & -- & 1905 & -- & 1944 & 133 & 1983 & 125 \\
\hline 1828 & -- & 1867 & -- & 1906 & -- & 1945 & 104 & 1984 & 129 \\
\hline 1829 & -- & 1868 & -- & 1907 & -- & 1946 & 133 & 1985 & 128 \\
\hline 1830 & -- & 1869 & -- & 1908 & -- & 1947 & 140 & 1986 & 120 \\
\hline 1831 & -- & 1870 & -- & 1909 & -- & 1948 & 127 & 1987 & 109 \\
\hline 1832 & -- & 1871 & -- & 1910 & -- & 1949 & 123 & 1988 & 123 \\
\hline 1833 & -- & 1872 & -- & 1911 & -- & 1950 & 128 & 1989 & 125 \\
\hline 1834 & -- & 1873 & -- & 1912 & -- & 1951 & 117 & 1990 & 122 \\
\hline 1835 & -- & 1874 & -- & 1913 & -- & 1952 & 128 & 1991 & -- \\
\hline 1836 & -- & 1875 & -- & 1914 & -- & 1953 & 126 & 1992 & 134 \\
\hline 1837 & -- & 1876 & -- & 1915 & -- & 1954 & 123 & 1993 & 124 \\
\hline 1838 & -- & 1877 & -- & 1916 & -- & 1955 & 124 & 1994 & -- \\
\hline 1839 & -- & 1878 & -- & 1917 & -- & 1956 & 133 & 1995 & 125 \\
\hline 1840 & -- & 1879 & -- & 1918 & -- & 1957 & 128 & 1996 & -- \\
\hline 1841 & -- & 1880 & -- & 1919 & -- & 1958 & 115 & 1997 & 125 \\
\hline 1842 & -- & 1881 & -- & 1920 & -- & 1959 & 128 & 1998 & 121 \\
\hline 1843 & -- & 1882 & -- & 1921 & -- & 1960 & -- & 1999 & 124 \\
\hline 1844 & -- & 1883 & -- & 1922 & 115 & 1961 & -- & 2000 & 125 \\
\hline 1845 & -- & 1884 & -- & 1923 & 128 & 1962 & -- & & \\
\hline
\end{tabular}


Table 7. Ice-out dates for Embden Pond, Maine

$[--$, no data $]$

Location of Lake.-Lat. $44^{\circ} 56^{\prime} 20^{\prime \prime}$, long. $69^{\circ} 57^{\prime} 12^{\prime \prime}$.

Period of Record.-1925 to 2000.

Years of Record. - 76 .

Observer.-Maine Department of Inland Fisheries and Wildlife.

Earliest Ice-Out Date.-1981: 95.

Latest Ice-Out Date. -1972: 133.

\begin{tabular}{|c|c|c|c|c|c|c|c|c|c|}
\hline Year & $\begin{array}{c}\text { Julian } \\
\text { day }\end{array}$ & Year & $\begin{array}{c}\text { Julian } \\
\text { day }\end{array}$ & Year & $\begin{array}{c}\text { Julian } \\
\text { day }\end{array}$ & Year & $\begin{array}{c}\text { Julian } \\
\text { day }\end{array}$ & Year & $\begin{array}{c}\text { Julian } \\
\text { day }\end{array}$ \\
\hline 1807 & -- & 1846 & -- & 1885 & -- & 1924 & -- & 1963 & 114 \\
\hline 1808 & -- & 1847 & -- & 1886 & -- & 1925 & 109 & 1964 & 120 \\
\hline 1809 & -- & 1848 & -- & 1887 & -- & 1926 & 127 & 1965 & 119 \\
\hline 1810 & -- & 1849 & -- & 1888 & -- & 1927 & 108 & 1966 & 122 \\
\hline 1811 & -- & 1850 & -- & 1889 & -- & 1928 & 128 & 1967 & 122 \\
\hline 1812 & -- & 1851 & -- & 1890 & -- & 1929 & 120 & 1968 & 113 \\
\hline 1813 & -- & 1852 & -- & 1891 & -- & 1930 & 113 & 1969 & 124 \\
\hline 1814 & -- & 1853 & -- & 1892 & -- & 1931 & 100 & 1970 & 124 \\
\hline 1815 & -- & 1854 & -- & 1893 & -- & 1932 & 112 & 1971 & 128 \\
\hline 1816 & -- & 1855 & -- & 1894 & -- & 1933 & 120 & 1972 & 133 \\
\hline 1817 & -- & 1856 & -- & 1895 & -- & 1934 & 114 & 1973 & 115 \\
\hline 1818 & -- & 1857 & -- & 1896 & -- & 1935 & 117 & 1974 & 115 \\
\hline 1819 & -- & 1858 & -- & 1897 & -- & 1936 & 115 & 1975 & 124 \\
\hline 1820 & -- & 1859 & -- & 1898 & -- & 1937 & 122 & 1976 & 114 \\
\hline 1821 & -- & 1860 & -- & 1899 & -- & 1938 & 114 & 1977 & 114 \\
\hline 1822 & -- & 1861 & -- & 1900 & -- & 1939 & 129 & 1978 & 125 \\
\hline 1823 & -- & 1862 & -- & 1901 & -- & 1940 & 129 & 1979 & 110 \\
\hline 1824 & -- & 1863 & -- & 1902 & -- & 1941 & 107 & 1980 & 111 \\
\hline 1825 & -- & 1864 & -- & 1903 & -- & 1942 & 118 & 1981 & 95 \\
\hline 1826 & -- & 1865 & -- & 1904 & -- & 1943 & 128 & 1982 & 122 \\
\hline 1827 & -- & 1866 & -- & 1905 & -- & 1944 & 126 & 1983 & 108 \\
\hline 1828 & -- & 1867 & -- & 1906 & -- & 1945 & 102 & 1984 & 118 \\
\hline 1829 & -- & 1868 & -- & 1907 & -- & 1946 & 107 & 1985 & 111 \\
\hline 1830 & -- & 1869 & -- & 1908 & -- & 1947 & 116 & 1986 & 110 \\
\hline 1831 & -- & 1870 & -- & 1909 & -- & 1948 & 106 & 1987 & 105 \\
\hline 1832 & -- & 1871 & -- & 1910 & -- & 1949 & 106 & 1988 & 112 \\
\hline 1833 & -- & 1872 & -- & 1911 & -- & 1950 & 120 & 1989 & 110 \\
\hline 1834 & -- & 1873 & -- & 1912 & -- & 1951 & 109 & 1990 & 117 \\
\hline 1835 & -- & 1874 & -- & 1913 & -- & 1952 & 118 & 1991 & 115 \\
\hline 1836 & -- & 1875 & -- & 1914 & -- & 1953 & 105 & 1992 & 121 \\
\hline 1837 & -- & 1876 & -- & 1915 & -- & 1954 & 117 & 1993 & 121 \\
\hline 1838 & -- & 1877 & -- & 1916 & -- & 1955 & 111 & 1994 & 124 \\
\hline 1839 & -- & 1878 & -- & 1917 & -- & 1956 & 125 & 1995 & 112 \\
\hline 1840 & -- & 1879 & -- & 1918 & -- & 1957 & 113 & 1996 & 115 \\
\hline 1841 & -- & 1880 & -- & 1919 & -- & 1958 & 113 & 1997 & 118 \\
\hline 1842 & -- & 1881 & -- & 1920 & -- & 1959 & 119 & 1998 & 105 \\
\hline 1843 & -- & 1882 & -- & 1921 & -- & 1960 & 120 & 1999 & 104 \\
\hline 1844 & -- & 1883 & -- & 1922 & -- & 1961 & 126 & 2000 & 107 \\
\hline 1845 & -- & 1884 & -- & 1923 & -- & 1962 & 113 & & \\
\hline
\end{tabular}


Table 8. Ice-out dates for First Connecticut Lake, New Hampshire

[--, no data]

Location of Lake.-Lat. $45^{\circ} 05^{\prime} 18^{\prime \prime}$, long. $71^{\circ} 14^{\prime} 55^{\prime \prime}$.

Period of Record.-1920 to 2000.

Years of Record.- 81 .

Observers. - C. J. Carlson, PG\&E National Energy Group, Fred T. Scott.

Earliest Ice-Out Date.-1921: 99.

Latest Ice-Out Date.—1972: 140.

\begin{tabular}{|c|c|c|c|c|c|c|c|c|c|}
\hline Year & $\begin{array}{c}\text { Julian } \\
\text { day }\end{array}$ & Year & $\begin{array}{c}\text { Julian } \\
\text { day }\end{array}$ & Year & $\begin{array}{c}\text { Julian } \\
\text { day }\end{array}$ & Year & $\begin{array}{c}\text { Julian } \\
\text { day }\end{array}$ & Year & $\begin{array}{c}\text { Julian } \\
\text { day }\end{array}$ \\
\hline 1807 & -- & 1846 & -- & 1885 & -- & 1924 & 128 & 1963 & 126 \\
\hline 1808 & -- & 1847 & -- & 1886 & -- & 1925 & 113 & 1964 & 127 \\
\hline 1809 & -- & 1848 & -- & 1887 & -- & 1926 & 127 & 1965 & 128 \\
\hline 1810 & -- & 1849 & -- & 1888 & -- & 1927 & 113 & 1966 & 134 \\
\hline 1811 & -- & 1850 & -- & 1889 & -- & 1928 & 131 & 1967 & 128 \\
\hline 1812 & -- & 1851 & -- & 1890 & -- & 1929 & 129 & 1968 & 112 \\
\hline 1813 & -- & 1852 & -- & 1891 & -- & 1930 & 126 & 1969 & 131 \\
\hline 1814 & -- & 1853 & -- & 1892 & -- & 1931 & 105 & 1970 & 127 \\
\hline 1815 & -- & 1854 & -- & 1893 & -- & 1932 & 130 & 1971 & 132 \\
\hline 1816 & -- & 1855 & -- & 1894 & -- & 1933 & 127 & 1972 & 140 \\
\hline 1817 & -- & 1856 & -- & 1895 & -- & 1934 & 118 & 1973 & 120 \\
\hline 1818 & -- & 1857 & -- & 1896 & -- & 1935 & 130 & 1974 & 135 \\
\hline 1819 & -- & 1858 & -- & 1897 & -- & 1936 & 123 & 1975 & 132 \\
\hline 1820 & -- & 1859 & -- & 1898 & -- & 1937 & 128 & 1976 & 115 \\
\hline 1821 & -- & 1860 & -- & 1899 & -- & 1938 & 118 & 1977 & 126 \\
\hline 1822 & -- & 1861 & -- & 1900 & -- & 1939 & 131 & 1978 & 136 \\
\hline 1823 & -- & 1862 & -- & 1901 & -- & 1940 & 134 & 1979 & 119 \\
\hline 1824 & -- & 1863 & -- & 1902 & -- & 1941 & 115 & 1980 & 124 \\
\hline 1825 & -- & 1864 & -- & 1903 & -- & 1942 & 123 & 1981 & 118 \\
\hline 1826 & -- & 1865 & -- & 1904 & -- & 1943 & 138 & 1982 & 133 \\
\hline 1827 & -- & 1866 & -- & 1905 & -- & 1944 & 129 & 1983 & 124 \\
\hline 1828 & -- & 1867 & -- & 1906 & -- & 1945 & 103 & 1984 & 120 \\
\hline 1829 & -- & 1868 & -- & 1907 & -- & 1946 & 126 & 1985 & 129 \\
\hline 1830 & -- & 1869 & -- & 1908 & -- & 1947 & 137 & 1986 & 115 \\
\hline 1831 & -- & 1870 & -- & 1909 & -- & 1948 & 118 & 1987 & 110 \\
\hline 1832 & -- & 1871 & -- & 1910 & -- & 1949 & 113 & 1988 & 124 \\
\hline 1833 & -- & 1872 & -- & 1911 & -- & 1950 & 127 & 1989 & 132 \\
\hline 1834 & -- & 1873 & -- & 1912 & -- & 1951 & 119 & 1990 & 122 \\
\hline 1835 & -- & 1874 & -- & 1913 & -- & 1952 & 121 & 1991 & 121 \\
\hline 1836 & -- & 1875 & -- & 1914 & -- & 1953 & 104 & 1992 & 134 \\
\hline 1837 & -- & 1876 & -- & 1915 & -- & 1954 & 122 & 1993 & 122 \\
\hline 1838 & -- & 1877 & -- & 1916 & -- & 1955 & 118 & 1994 & 129 \\
\hline 1839 & -- & 1878 & -- & 1917 & -- & 1956 & 133 & 1995 & 125 \\
\hline 1840 & -- & 1879 & -- & 1918 & -- & 1957 & 121 & 1996 & 134 \\
\hline 1841 & -- & 1880 & -- & 1919 & -- & 1958 & 116 & 1997 & 132 \\
\hline 1842 & -- & 1881 & -- & 1920 & 128 & 1959 & 126 & 1998 & 108 \\
\hline 1843 & -- & 1882 & -- & 1921 & 99 & 1960 & 122 & 1999 & 124 \\
\hline 1844 & -- & 1883 & -- & 1922 & 123 & 1961 & 135 & 2000 & 124 \\
\hline 1845 & -- & 1884 & -- & 1923 & 127 & 1962 & 132 & & \\
\hline
\end{tabular}


Table 9. Ice-out dates for Houghtons (Hoosicwhisick) Pond, Massachusetts

$[--$, no data $]$

Location of Lake.-Lat. $42^{\circ} 12^{\prime} 26^{\prime \prime}$, long. $71^{\circ} 05^{\prime} 47^{\prime \prime}$.

Period of Record.-1886 to 2000.

Years of Record. -115 .

Observer.-Blue Hills Observatory.

Earliest Ice-Out Date.-1981: 53.

Latest Ice-Out Date.-1926: 104.

\begin{tabular}{|c|c|c|c|c|c|c|c|c|c|}
\hline Year & $\begin{array}{c}\text { Julian } \\
\text { day }\end{array}$ & Year & $\begin{array}{c}\text { Julian } \\
\text { day }\end{array}$ & Year & $\begin{array}{c}\text { Julian } \\
\text { day }\end{array}$ & Year & $\begin{array}{c}\text { Julian } \\
\text { day }\end{array}$ & Year & $\begin{array}{c}\text { Julian } \\
\text { day }\end{array}$ \\
\hline 1807 & -- & 1846 & -- & 1885 & -- & 1924 & 97 & 1963 & 87 \\
\hline 1808 & -- & 1847 & -- & 1886 & 86 & 1925 & 71 & 1964 & 85 \\
\hline 1809 & -- & 1848 & -- & 1887 & 100 & 1926 & 104 & 1965 & 76 \\
\hline 1810 & -- & 1849 & -- & 1888 & 100 & 1927 & 78 & 1966 & 83 \\
\hline 1811 & -- & 1850 & -- & 1889 & 71 & 1928 & 87 & 1967 & 94 \\
\hline 1812 & -- & 1851 & -- & 1890 & 71 & 1929 & 80 & 1968 & 89 \\
\hline 1813 & -- & 1852 & -- & 1891 & 81 & 1930 & 68 & 1969 & 95 \\
\hline 1814 & -- & 1853 & -- & 1892 & 94 & 1931 & 86 & 1970 & 80 \\
\hline 1815 & -- & 1854 & -- & 1893 & 91 & 1932 & 89 & 1971 & 90 \\
\hline 1816 & -- & 1855 & -- & 1894 & 71 & 1933 & 86 & 1972 & 89 \\
\hline 1817 & -- & 1856 & -- & 1895 & 95 & 1934 & 91 & 1973 & 66 \\
\hline 1818 & -- & 1857 & -- & 1896 & 93 & 1935 & 85 & 1974 & 63 \\
\hline 1819 & -- & 1858 & -- & 1897 & 83 & 1936 & 81 & 1975 & 75 \\
\hline 1820 & -- & 1859 & -- & 1898 & 74 & 1937 & 77 & 1976 & 60 \\
\hline 1821 & -- & 1860 & -- & 1899 & 97 & 1938 & 77 & 1977 & 83 \\
\hline 1822 & -- & 1861 & -- & 1900 & 80 & 1939 & 91 & 1978 & 92 \\
\hline 1823 & -- & 1862 & -- & 1901 & 84 & 1940 & 97 & 1979 & 84 \\
\hline 1824 & -- & 1863 & -- & 1902 & 76 & 1941 & 95 & 1980 & 78 \\
\hline 1825 & -- & 1864 & -- & 1903 & 71 & 1942 & 68 & 1981 & 53 \\
\hline 1826 & -- & 1865 & -- & 1904 & 95 & 1943 & 82 & 1982 & 80 \\
\hline 1827 & -- & 1866 & -- & 1905 & 92 & 1944 & 86 & 1983 & 70 \\
\hline 1828 & -- & 1867 & -- & 1906 & 94 & 1945 & 79 & 1984 & 56 \\
\hline 1829 & -- & 1868 & -- & 1907 & 87 & 1946 & 73 & 1985 & 65 \\
\hline 1830 & -- & 1869 & -- & 1908 & 86 & 1947 & 74 & 1986 & 72 \\
\hline 1831 & -- & 1870 & -- & 1909 & 76 & 1948 & 85 & 1987 & 90 \\
\hline 1832 & -- & 1871 & -- & 1910 & 83 & 1949 & 64 & 1988 & 84 \\
\hline 1833 & -- & 1872 & -- & 1911 & 88 & 1950 & 89 & 1989 & 76 \\
\hline 1834 & -- & 1873 & -- & 1912 & 90 & 1951 & 60 & 1990 & 54 \\
\hline 1835 & -- & 1874 & -- & 1913 & 73 & 1952 & 85 & 1991 & 62 \\
\hline 1836 & -- & 1875 & -- & 1914 & 92 & 1953 & 58 & 1992 & 68 \\
\hline 1837 & -- & 1876 & -- & 1915 & 74 & 1954 & 57 & 1993 & 98 \\
\hline 1838 & -- & 1877 & -- & 1916 & 97 & 1955 & 59 & 1994 & 95 \\
\hline 1839 & -- & 1878 & -- & 1917 & 96 & 1956 & 98 & 1995 & 73 \\
\hline 1840 & -- & 1879 & -- & 1918 & 92 & 1957 & 71 & 1996 & 84 \\
\hline 1841 & -- & 1880 & -- & 1919 & 65 & 1958 & 73 & 1997 & 54 \\
\hline 1842 & -- & 1881 & -- & 1920 & 96 & 1959 & 79 & 1998 & 55 \\
\hline 1843 & -- & 1882 & -- & 1921 & 72 & 1960 & 55 & 1999 & 63 \\
\hline 1844 & -- & 1883 & -- & 1922 & 87 & 1961 & 91 & 2000 & 68 \\
\hline 1845 & -- & 1884 & -- & 1923 & 99 & 1962 & 86 & & \\
\hline
\end{tabular}


Table 10. Ice-out dates for Kezar Lake, Maine

$[--$, no data $]$

Location of Lake.-Lat. $44^{\circ} 11^{\prime} 04^{\prime \prime}$, long. $70^{\circ} 54^{\prime} 12^{\prime \prime}$.

Period of Record.-1901 to 1999.

Years of Record.-99.

Observers.-Lovell Town Office, Advertiser Democrat, Arthur P. Stone.

Earliest Ice-Out Date.—1921: 93.

Latest Ice-Out Date.-1928, 1939, 1940: 126.

\begin{tabular}{|c|c|c|c|c|c|c|c|c|c|}
\hline Year & $\begin{array}{c}\text { Julian } \\
\text { day }\end{array}$ & Year & $\begin{array}{c}\text { Julian } \\
\text { day }\end{array}$ & Year & $\begin{array}{c}\text { Julian } \\
\text { day }\end{array}$ & Year & $\begin{array}{c}\text { Julian } \\
\text { day }\end{array}$ & Year & $\begin{array}{c}\text { Julian } \\
\text { day }\end{array}$ \\
\hline 1807 & -- & 1846 & -- & 1885 & -- & 1924 & 113 & 1963 & 113 \\
\hline 1808 & -- & 1847 & -- & 1886 & -- & 1925 & 105 & 1964 & 119 \\
\hline 1809 & -- & 1848 & -- & 1887 & -- & 1926 & 123 & 1965 & 118 \\
\hline 1810 & -- & 1849 & -- & 1888 & -- & 1927 & 106 & 1966 & 113 \\
\hline 1811 & -- & 1850 & -- & 1889 & -- & 1928 & 126 & 1967 & 118 \\
\hline 1812 & -- & 1851 & -- & 1890 & -- & 1929 & 115 & 1968 & 107 \\
\hline 1813 & -- & 1852 & -- & 1891 & -- & 1930 & 104 & 1969 & 119 \\
\hline 1814 & -- & 1853 & -- & 1892 & -- & 1931 & 103 & 1970 & 123 \\
\hline 1815 & -- & 1854 & -- & 1893 & -- & 1932 & 119 & 1971 & 122 \\
\hline 1816 & -- & 1855 & -- & 1894 & -- & 1933 & 119 & 1972 & 124 \\
\hline 1817 & -- & 1856 & -- & 1895 & -- & 1934 & 114 & 1973 & 109 \\
\hline 1818 & -- & 1857 & -- & 1896 & -- & 1935 & 115 & 1974 & 116 \\
\hline 1819 & -- & 1858 & -- & 1897 & -- & 1936 & 113 & 1975 & 121 \\
\hline 1820 & -- & 1859 & -- & 1898 & -- & 1937 & 122 & 1976 & 110 \\
\hline 1821 & -- & 1860 & -- & 1899 & -- & 1938 & 110 & 1977 & 110 \\
\hline 1822 & -- & 1861 & -- & 1900 & -- & 1939 & 126 & 1978 & 122 \\
\hline 1823 & -- & 1862 & -- & 1901 & 110 & 1940 & 126 & 1979 & 116 \\
\hline 1824 & -- & 1863 & -- & 1902 & 103 & 1941 & 104 & 1980 & 111 \\
\hline 1825 & -- & 1864 & -- & 1903 & 109 & 1942 & 114 & 1981 & 97 \\
\hline 1826 & -- & 1865 & -- & 1904 & 123 & 1943 & 122 & 1982 & 120 \\
\hline 1827 & -- & 1866 & -- & 1905 & 112 & 1944 & 123 & 1983 & 104 \\
\hline 1828 & -- & 1867 & -- & 1906 & 98 & 1945 & 99 & 1984 & 112 \\
\hline 1829 & -- & 1868 & -- & 1907 & 120 & 1946 & 100 & 1985 & 107 \\
\hline 1830 & -- & 1869 & -- & 1908 & 120 & 1947 & 114 & 1986 & 107 \\
\hline 1831 & -- & 1870 & -- & 1909 & 117 & 1948 & 105 & 1987 & 103 \\
\hline 1832 & -- & 1871 & -- & 1910 & 97 & 1949 & 102 & 1988 & 108 \\
\hline 1833 & -- & 1872 & -- & 1911 & 121 & 1950 & 117 & 1989 & 118 \\
\hline 1834 & -- & 1873 & -- & 1912 & 116 & 1951 & 110 & 1990 & 113 \\
\hline 1835 & -- & 1874 & -- & 1913 & 109 & 1952 & 114 & 1991 & 101 \\
\hline 1836 & -- & 1875 & -- & 1914 & 116 & 1953 & 104 & 1992 & 116 \\
\hline 1837 & -- & 1876 & -- & 1915 & 107 & 1954 & 112 & 1993 & 118 \\
\hline 1838 & -- & 1877 & -- & 1916 & 113 & 1955 & 112 & 1994 & 118 \\
\hline 1839 & -- & 1878 & -- & 1917 & 118 & 1956 & 122 & 1995 & 114 \\
\hline 1840 & -- & 1879 & -- & 1918 & 113 & 1957 & 107 & 1996 & 111 \\
\hline 1841 & -- & 1880 & -- & 1919 & 100 & 1958 & 110 & 1997 & 107 \\
\hline 1842 & -- & 1881 & -- & 1920 & 120 & 1959 & 113 & 1998 & 114 \\
\hline 1843 & -- & 1882 & -- & 1921 & 93 & 1960 & 115 & 1999 & 103 \\
\hline 1844 & -- & 1883 & -- & 1922 & 107 & 1961 & 121 & 2000 & -- \\
\hline 1845 & -- & 1884 & -- & 1923 & 115 & 1962 & 114 & & \\
\hline
\end{tabular}


Table 11. Ice-out dates for Maranacook Lake, Maine

$[--$, no data $]$

Location of Lake.-Lat. $44^{\circ} 20^{\prime} 03^{\prime \prime}$, long. $69^{\circ} 57^{\prime} 21^{\prime \prime}$.

Period of Record.-1925 to 2000.

Years of Record.-76.

Observers and Ice-Out Definition.-Community Advertiser, John Neff, Roscoe Sprague, Roger Audette. Ice-out for most years thought to be observed from southern end of lake.

Earliest Ice-Out Date.-1981: 84.

Latest Ice-Out Date.-1939: 124.

\begin{tabular}{|c|c|c|c|c|c|c|c|c|c|}
\hline Year & $\begin{array}{c}\text { Julian } \\
\text { day }\end{array}$ & Year & $\begin{array}{c}\text { Julian } \\
\text { day }\end{array}$ & Year & $\begin{array}{l}\text { Julian } \\
\text { day }\end{array}$ & Year & $\begin{array}{c}\text { Julian } \\
\text { day }\end{array}$ & Year & $\begin{array}{c}\text { Julian } \\
\text { day }\end{array}$ \\
\hline 1807 & -- & 1846 & -- & 1885 & -- & 1924 & -- & 1963 & 111 \\
\hline 1808 & -- & 1847 & -- & 1886 & -- & 1925 & 105 & 1964 & 112 \\
\hline 1809 & -- & 1848 & -- & 1887 & -- & 1926 & 123 & 1965 & 114 \\
\hline 1810 & -- & 1849 & -- & 1888 & -- & 1927 & 120 & 1966 & 108 \\
\hline 1811 & -- & 1850 & -- & 1889 & -- & 1928 & 111 & 1967 & 118 \\
\hline 1812 & -- & 1851 & -- & 1890 & -- & 1929 & 110 & 1968 & 104 \\
\hline 1813 & -- & 1852 & -- & 1891 & -- & 1930 & 101 & 1969 & 115 \\
\hline 1814 & -- & 1853 & -- & 1892 & -- & 1931 & 101 & 1970 & 119 \\
\hline 1815 & -- & 1854 & -- & 1893 & -- & 1932 & 110 & 1971 & 123 \\
\hline 1816 & -- & 1855 & -- & 1894 & -- & 1933 & 109 & 1972 & 123 \\
\hline 1817 & -- & 1856 & -- & 1895 & -- & 1934 & 110 & 1973 & 107 \\
\hline 1818 & -- & 1857 & -- & 1896 & -- & 1935 & 107 & 1974 & 100 \\
\hline 1819 & -- & 1858 & -- & 1897 & -- & 1936 & 105 & 1975 & 118 \\
\hline 1820 & -- & 1859 & --. & 1898 & -- & 1937 & 116 & 1976 & 106 \\
\hline 1821 & -- & 1860 & -- & 1899 & -- & 1938 & 109 & 1977 & 108 \\
\hline 1822 & -- & 1861 & --- & 1900 & -- & 1939 & 124 & 1978 & 118 \\
\hline 1823 & -- & 1862 & -- & 1901 & -- & 1940 & 123 & 1979 & 107 \\
\hline 1824 & -- & 1863 & -- & 1902 & -- & 1941 & 102 & 1980 & 100 \\
\hline 1825 & -- & 1864 & -- & 1903 & -- & 1942 & 110 & 1981 & 84 \\
\hline 1826 & -- & 1865 & -- & 1904 & -- & 1943 & 116 & 1982 & 115 \\
\hline 1827 & -- & 1866 & -- & 1905 & -- & 1944 & 119 & 1983 & 92 \\
\hline 1828 & -- & 1867 & -- & 1906 & -- & 1945 & 104 & 1984 & 109 \\
\hline 1829 & -- & 1868 & -- & 1907 & -- & 1946 & 101 & 1985 & 97 \\
\hline 1830 & -- & 1869 & -- & 1908 & -- & 1947 & 109 & 1986 & 102 \\
\hline 1831 & -- & 1870 & -- & 1909 & -- & 1948 & 100 & 1987 & 102 \\
\hline 1832 & -- & 1871 & -- & 1910 & -- & 1949 & 100 & 1988 & 101 \\
\hline 1833 & -- & 1872 & -- & 1911 & -- & 1950 & 111 & 1989 & 114 \\
\hline 1834 & -- & 1873 & --- & 1912 & -- & 1951 & 97 & 1990 & 103 \\
\hline 1835 & -- & 1874 & -- & 1913 & -- & 1952 & 111 & 1991 & 97 \\
\hline 1836 & -- & 1875 & -- & 1914 & -- & 1953 & 89 & 1992 & 100 \\
\hline 1837 & -- & 1876 & -- & 1915 & -- & 1954 & 110 & 1993 & 110 \\
\hline 1838 & -- & 1877 & -- & 1916 & -- & 1955 & 105 & 1994 & 113 \\
\hline 1839 & -- & 1878 & -- & 1917 & -- & 1956 & 119 & 1995 & 100 \\
\hline 1840 & -- & 1879 & -- & 1918 & -- & 1957 & 105 & 1996 & 96 \\
\hline 1841 & -- & 1880 & -- & 1919 & -- & 1958 & 106 & 1997 & 115 \\
\hline 1842 & -- & 1881 & --. & 1920 & -- & 1959 & 111 & 1998 & 100 \\
\hline 1843 & -- & 1882 & -- & 1921 & -- & 1960 & 113 & 1999 & 99 \\
\hline 1844 & -- & 1883 & -- & 1922 & -- & 1961 & 121 & 2000 & 100 \\
\hline 1845 & -- & 1884 & -- & 1923 & -- & 1962 & 111 & & \\
\hline
\end{tabular}


Table 12. Ice-out dates for Messalonskee Lake, Maine

$[--$, no data $]$

Location of Lake.-Lat. $44^{\circ} 28^{\prime} 41^{\prime \prime}$, long. $69^{\circ} 47^{\prime} 34^{\prime \prime}$.

Period of Record.-1909 to 2000.

Years of Record.- 67.

Observers.-Central Maine Morning Sentinel, Joyce Rushton.

Earliest Ice-Out Date.-1985: 90.

Latest Ice-Out Date.-1940: 128.

\begin{tabular}{|c|c|c|c|c|c|c|c|c|c|}
\hline Year & $\begin{array}{c}\text { Julian } \\
\text { day }\end{array}$ & Year & $\begin{array}{c}\text { Julian } \\
\text { day }\end{array}$ & Year & $\begin{array}{c}\text { Julian } \\
\text { day }\end{array}$ & Year & $\begin{array}{c}\text { Julian } \\
\text { day }\end{array}$ & Year & $\begin{array}{c}\text { Julian } \\
\text { day }\end{array}$ \\
\hline 1807 & -- & 1846 & -- & 1885 & -- & 1924 & 111 & 1963 & -- \\
\hline 1808 & -- & 1847 & -- & 1886 & -- & 1925 & 100 & 1964 & -- \\
\hline 1809 & -- & 1848 & -- & 1887 & -- & 1926 & 123 & 1965 & -- \\
\hline 1810 & -- & 1849 & -- & 1888 & -- & 1927 & 106 & 1966 & -- \\
\hline 1811 & -- & 1850 & -- & 1889 & -- & 1928 & 120 & 1967 & -- \\
\hline 1812 & -- & 1851 & -- & 1890 & -- & 1929 & 115 & 1968 & -- \\
\hline 1813 & -- & 1852 & -- & 1891 & -- & 1930 & 113 & 1969 & -- \\
\hline 1814 & -- & 1853 & -- & 1892 & -- & 1931 & 105 & 1970 & -- \\
\hline 1815 & -- & 1854 & -- & 1893 & -- & 1932 & 113 & 1971 & -- \\
\hline 1816 & -- & 1855 & -- & 1894 & -- & 1933 & 115 & 1972 & -- \\
\hline 1817 & -- & 1856 & -- & 1895 & -- & 1934 & 114 & 1973 & -- \\
\hline 1818 & -- & 1857 & -- & 1896 & -- & 1935 & 116 & 1974 & -- \\
\hline 1819 & -- & 1858 & -- & 1897 & -- & 1936 & 107 & 1975 & -- \\
\hline 1820 & -- & 1859 & -- & 1898 & -- & 1937 & 117 & 1976 & -- \\
\hline 1821 & -- & 1860 & -- & 1899 & -- & 1938 & 110 & 1977 & -- \\
\hline 1822 & -- & 1861 & -- & 1900 & -- & 1939 & 127 & 1978 & -- \\
\hline 1823 & -- & 1862 & -- & 1901 & -- & 1940 & 128 & 1979 & -- \\
\hline 1824 & -- & 1863 & -- & 1902 & -- & 1941 & 106 & 1980 & -- \\
\hline 1825 & -- & 1864 & -- & 1903 & -- & 1942 & 112 & 1981 & -- \\
\hline 1826 & -- & 1865 & -- & 1904 & -- & 1943 & 119 & 1982 & -- \\
\hline 1827 & -- & 1866 & -- & 1905 & -- & 1944 & 119 & 1983 & -- \\
\hline 1828 & -- & 1867 & -- & 1906 & -- & 1945 & -- & 1984 & -- \\
\hline 1829 & -- & 1868 & -- & 1907 & -- & 1946 & 105 & 1985 & 90 \\
\hline 1830 & -- & 1869 & -- & 1908 & -- & 1947 & 110 & 1986 & -- \\
\hline 1831 & -- & 1870 & -- & 1909 & 114 & 1948 & 107 & 1987 & 102 \\
\hline 1832 & -- & 1871 & -- & 1910 & 95 & 1949 & 102 & 1988 & 101 \\
\hline 1833 & -- & 1872 & -- & 1911 & 120 & 1950 & 113 & 1989 & 116 \\
\hline 1834 & -- & 1873 & -- & 1912 & 115 & 1951 & 110 & 1990 & 109 \\
\hline 1835 & -- & 1874 & -- & 1913 & 110 & 1952 & 114 & 1991 & 105 \\
\hline 1836 & -- & 1875 & -- & 1914 & 120 & 1953 & 100 & 1992 & 113 \\
\hline 1837 & -- & 1876 & -- & 1915 & 105 & 1954 & 113 & 1993 & 113 \\
\hline 1838 & -- & 1877 & -- & 1916 & -- & 1955 & 108 & 1994 & 116 \\
\hline 1839 & -- & 1878 & -- & 1917 & 118 & 1956 & 122 & 1995 & 106 \\
\hline 1840 & -- & 1879 & -- & 1918 & 114 & 1957 & 108 & 1996 & 108 \\
\hline 1841 & -- & 1880 & -- & 1919 & 103 & 1958 & 108 & 1997 & 116 \\
\hline 1842 & -- & 1881 & -- & 1920 & 121 & 1959 & 114 & 1998 & 100 \\
\hline 1843 & -- & 1882 & -- & 1921 & 97 & 1960 & 119 & 1999 & 99 \\
\hline 1844 & -- & 1883 & -- & 1922 & 105 & 1961 & 123 & 2000 & 99 \\
\hline 1845 & -- & 1884 & -- & 1923 & 115 & 1962 & 113 & & \\
\hline
\end{tabular}


Table 13. Ice-out dates for Moosehead Lake, Maine

[--, no data]

Location of Lake.-Lat. $45^{\circ} 38^{\prime} 27^{\prime \prime}$, long. 69 39'57".

Period of Record.-1848 to 2000.

Years of Record. - 153 .

Observers and Ice-Out Definition. - Kennebec Water Power Company, Maine Department of Inland Fisheries and Wildlife. Ice-out definition for most years thought be date of open navigation from Greenville to Northeast Carry.

Earliest Ice-Out Date.-1945: 104.

Latest Ice-Out Date.-1878: 149.

\begin{tabular}{|c|c|c|c|c|c|c|c|c|c|}
\hline Year & $\begin{array}{c}\text { Julian } \\
\text { day }\end{array}$ & Year & $\begin{array}{c}\text { Julian } \\
\text { day }\end{array}$ & Year & $\begin{array}{c}\text { Julian } \\
\text { day }\end{array}$ & Year & $\begin{array}{c}\text { Julian } \\
\text { day }\end{array}$ & Year & $\begin{array}{c}\text { Julian } \\
\text { day }\end{array}$ \\
\hline 1807 & -- & 1846 & -- & 1885 & 136 & 1924 & 130 & 1963 & 127 \\
\hline 1808 & -- & 1847 & -- & 1886 & 122 & 1925 & 118 & 1964 & 128 \\
\hline 1809 & -- & 1848 & 121 & 1887 & 133 & 1926 & 138 & 1965 & 127 \\
\hline 1810 & -- & 1849 & 132 & 1888 & 143 & 1927 & 115 & 1966 & 126 \\
\hline 1811 & -- & 1850 & 129 & 1889 & 120 & 1928 & 133 & 1967 & 128 \\
\hline 1812 & -- & 1851 & 134 & 1890 & 129 & 1929 & 128 & 1968 & 116 \\
\hline 1813 & -- & 1852 & 138 & 1891 & 134 & 1930 & 125 & 1969 & 133 \\
\hline 1814 & -- & 1853 & 131 & 1892 & 125 & 1931 & 114 & 1970 & 136 \\
\hline 1815 & -- & 1854 & 140 & 1893 & 138 & 1932 & 126 & 1971 & 134 \\
\hline 1816 & -- & 1855 & 137 & 1894 & 121 & 1933 & 124 & 1972 & 143 \\
\hline 1817 & -- & 1856 & 130 & 1895 & 126 & 1934 & 124 & 1973 & 125 \\
\hline 1818 & -- & 1857 & 132 & 1896 & 129 & 1935 & 130 & 1974 & 136 \\
\hline 1819 & -- & 1858 & 133 & 1897 & 128 & 1936 & 123 & 1975 & 132 \\
\hline 1820 & -- & 1859 & 134 & 1898 & 124 & 1937 & 130 & 1976 & 122 \\
\hline 1821 & -- & 1860 & 132 & 1899 & 127 & 1938 & 119 & 1977 & 126 \\
\hline 1822 & -- & 1861 & 132 & 1900 & 131 & 1939 & 137 & 1978 & 135 \\
\hline 1823 & -- & 1862 & 138 & 1901 & 119 & 1940 & 138 & 1979 & 121 \\
\hline 1824 & -- & 1863 & 138 & 1902 & 118 & 1941 & 119 & 1980 & 123 \\
\hline 1825 & -- & 1864 & 127 & 1903 & 118 & 1942 & 125 & 1981 & 117 \\
\hline 1826 & -- & 1865 & 124 & 1904 & 131 & 1943 & 138 & 1982 & 133 \\
\hline 1827 & -- & 1866 & 131 & 1905 & 122 & 1944 & 135 & 1983 & 122 \\
\hline 1828 & -- & 1867 & 139 & 1906 & 133 & 1945 & 104 & 1984 & 127 \\
\hline 1829 & -- & 1868 & 139 & 1907 & 134 & 1946 & 126 & 1985 & 123 \\
\hline 1830 & -- & 1869 & 130 & 1908 & 132 & 1947 & 133 & 1986 & 118 \\
\hline 1831 & -- & 1870 & 124 & 1909 & 135 & 1948 & 125 & 1987 & 111 \\
\hline 1832 & -- & 1871 & 133 & 1910 & 110 & 1949 & 113 & 1988 & 124 \\
\hline 1833 & -- & 1872 & 132 & 1911 & 133 & 1950 & 127 & 1989 & 132 \\
\hline 1834 & -- & 1873 & 136 & 1912 & 129 & 1951 & 119 & 1990 & 127 \\
\hline 1835 & -- & 1874 & 146 & 1913 & 122 & 1952 & 121 & 1991 & 122 \\
\hline 1836 & -- & 1875 & 144 & 1914 & 135 & 1953 & 114 & 1992 & 134 \\
\hline 1837 & -- & 1876 & 144 & 1915 & 121 & 1954 & 122 & 1993 & 128 \\
\hline 1838 & -- & 1877 & 126 & 1916 & 125 & 1955 & 124 & 1994 & 131 \\
\hline 1839 & -- & 1878 & 149 & 1917 & 134 & 1956 & 136 & 1995 & 122 \\
\hline 1840 & -- & 1879 & 134 & 1918 & 121 & 1957 & 122 & 1996 & 126 \\
\hline 1841 & -- & 1880 & 127 & 1919 & 123 & 1958 & 122 & 1997 & 131 \\
\hline 1842 & -- & 1881 & 129 & 1920 & 135 & 1959 & 129 & 1998 & 117 \\
\hline 1843 & -- & 1882 & 138 & 1921 & 112 & 1960 & 127 & 1999 & 120 \\
\hline 1844 & -- & 1883 & 133 & 1922 & 121 & 1961 & 137 & 2000 & 121 \\
\hline 1845 & -- & 1884 & 129 & 1923 & 130 & 1962 & 138 & & \\
\hline
\end{tabular}


Table 14. Ice-out dates for Mooselookmeguntic Lake, Maine

[--, no data]

Location of Lake.-Lat. $44^{\circ} 54^{\prime} 25^{\prime \prime}$, long. $70^{\circ} 48^{\prime} 40^{\prime \prime}$.

Period of Record.-1884 to 2000.

Years of Record.-99.

Observers.-Union Water Power Company, FPL Energy Maine.

Earliest Ice-Out Date.-1945: 104.

Latest Ice-Out Date.—1972: 143.

\begin{tabular}{|c|c|c|c|c|c|c|c|c|c|}
\hline Year & $\begin{array}{c}\text { Julian } \\
\text { day }\end{array}$ & Year & $\begin{array}{c}\text { Julian } \\
\text { day }\end{array}$ & Year & $\begin{array}{c}\text { Julian } \\
\text { day }\end{array}$ & Year & $\begin{array}{c}\text { Julian } \\
\text { day }\end{array}$ & Year & $\begin{array}{c}\text { Julian } \\
\text { day }\end{array}$ \\
\hline 1807 & -- & 1846 & -- & 1885 & 130 & 1924 & 132 & 1963 & 125 \\
\hline 1808 & -- & 1847 & -- & 1886 & -- & 1925 & 118 & 1964 & 127 \\
\hline 1809 & -- & 1848 & -- & 1887 & -- & 1926 & 136 & 1965 & 127 \\
\hline 1810 & -- & 1849 & -- & 1888 & -- & 1927 & 117 & 1966 & 136 \\
\hline 1811 & -- & 1850 & -- & 1889 & -- & 1928 & 134 & 1967 & 129 \\
\hline 1812 & -- & 1851 & -- & 1890 & 131 & 1929 & 128 & 1968 & 114 \\
\hline 1813 & -- & 1852 & -- & 1891 & 130 & 1930 & 126 & 1969 & 132 \\
\hline 1814 & -- & 1853 & -- & 1892 & 127 & 1931 & 116 & 1970 & 136 \\
\hline 1815 & -- & 1854 & -- & 1893 & 141 & 1932 & 127 & 1971 & 133 \\
\hline 1816 & -- & 1855 & -- & 1894 & -- & 1933 & 127 & 1972 & 143 \\
\hline 1817 & -- & 1856 & -- & 1895 & -- & 1934 & -- & 1973 & 124 \\
\hline 1818 & -- & 1857 & -- & 1896 & -- & 1935 & -- & 1974 & 135 \\
\hline 1819 & -- & 1858 & -- & 1897 & 133 & 1936 & 125 & 1975 & 133 \\
\hline 1820 & -- & 1859 & -- & 1898 & 124 & 1937 & 133 & 1976 & 120 \\
\hline 1821 & -- & 1860 & -- & 1899 & 128 & 1938 & 118 & 1977 & 125 \\
\hline 1822 & -- & 1861 & -- & 1900 & -- & 1939 & 135 & 1978 & 136 \\
\hline 1823 & -- & 1862 & -- & 1901 & -- & 1940 & 138 & 1979 & 122 \\
\hline 1824 & -- & 1863 & -- & 1902 & -- & 1941 & 117 & 1980 & 124 \\
\hline 1825 & -- & 1864 & -- & 1903 & -- & 1942 & 125 & 1981 & 119 \\
\hline 1826 & -- & 1865 & -- & 1904 & -- & 1943 & 140 & 1982 & 134 \\
\hline 1827 & -- & 1866 & -- & 1905 & -- & 1944 & 131 & 1983 & 127 \\
\hline 1828 & -- & 1867 & -- & 1906 & 133 & 1945 & 104 & 1984 & 123 \\
\hline 1829 & -- & 1868 & -- & 1907 & -- & 1946 & 121 & 1985 & 128 \\
\hline 1830 & -- & 1869 & -- & 1908 & -- & 1947 & 134 & 1986 & 116 \\
\hline 1831 & -- & 1870 & -- & 1909 & -- & 1948 & 122 & 1987 & 111 \\
\hline 1832 & -- & 1871 & -- & 1910 & 108 & 1949 & 118 & 1988 & 124 \\
\hline 1833 & -- & 1872 & -- & 1911 & 130 & 1950 & 128 & 1989 & 130 \\
\hline 1834 & -- & 1873 & -- & 1912 & 127 & 1951 & 122 & 1990 & 125 \\
\hline 1835 & -- & 1874 & -- & 1913 & 124 & 1952 & 123 & 1991 & 121 \\
\hline 1836 & -- & 1875 & -- & 1914 & 135 & 1953 & 113 & 1992 & 134 \\
\hline 1837 & -- & 1876 & -- & 1915 & 119 & 1954 & 127 & 1993 & 123 \\
\hline 1838 & -- & 1877 & -- & 1916 & 129 & 1955 & 120 & 1994 & 130 \\
\hline 1839 & -- & 1878 & -- & 1917 & 137 & 1956 & 135 & 1995 & 125 \\
\hline 1840 & -- & 1879 & -- & 1918 & 124 & 1957 & 120 & 1996 & 131 \\
\hline 1841 & -- & 1880 & -- & 1919 & 123 & 1958 & 122 & 1997 & 131 \\
\hline 1842 & -- & 1881 & -- & 1920 & 139 & 1959 & 127 & 1998 & 112 \\
\hline 1843 & -- & 1882 & -- & 1921 & 105 & 1960 & 126 & 1999 & 123 \\
\hline 1844 & -- & 1883 & -- & 1922 & 125 & 1961 & 135 & 2000 & 121 \\
\hline 1845 & -- & 1884 & 132 & 1923 & 129 & 1962 & 131 & & \\
\hline
\end{tabular}


Table 15. Ice-out dates for Pennesseewassee (Norway) Lake, Maine

$[--$, no data $]$

Location of Lake.-Lat. 44 13'34", long. 70 34'43".

Period of Record.-1874 to 1999.

Years of Record. - 125 .

Observer.-Woodman's Sporting Goods.

Earliest Ice-Out Date.-1981: 91.

Latest Ice-Out Date.-1874: 133.

\begin{tabular}{|c|c|c|c|c|c|c|c|c|c|}
\hline Year & $\begin{array}{c}\text { Julian } \\
\text { day }\end{array}$ & Year & $\begin{array}{c}\text { Julian } \\
\text { day }\end{array}$ & Year & $\begin{array}{c}\text { Julian } \\
\text { day }\end{array}$ & Year & $\begin{array}{c}\text { Julian } \\
\text { day }\end{array}$ & Year & $\begin{array}{c}\text { Julian } \\
\text { day }\end{array}$ \\
\hline 1807 & -- & 1846 & -- & 1885 & 122 & 1924 & 112 & 1963 & 111 \\
\hline 1808 & -- & 1847 & -- & 1886 & 114 & 1925 & 104 & 1964 & 118 \\
\hline 1809 & -- & 1848 & -- & 1887 & 126 & 1926 & 123 & 1965 & 114 \\
\hline 1810 & -- & 1849 & -- & 1888 & 131 & 1927 & 106 & 1966 & 113 \\
\hline 1811 & -- & 1850 & -- & 1889 & 106 & 1928 & 118 & 1967 & 118 \\
\hline 1812 & -- & 1851 & -- & 1890 & 116 & 1929 & 116 & 1968 & 105 \\
\hline 1813 & -- & 1852 & -- & 1891 & 118 & 1930 & 104 & 1969 & 117 \\
\hline 1814 & -- & 1853 & -- & 1892 & 109 & 1931 & 102 & 1970 & 121 \\
\hline 1815 & -- & 1854 & -- & 1893 & 128 & 1932 & 112 & 1971 & 123 \\
\hline 1816 & -- & 1855 & -- & 1894 & 114 & 1933 & 118 & 1972 & 126 \\
\hline 1817 & -- & 1856 & -- & 1895 & 111 & 1934 & 113 & 1973 & 112 \\
\hline 1818 & -- & 1857 & -- & 1896 & 118 & 1935 & 115 & 1974 & 111 \\
\hline 1819 & -- & 1858 & -- & 1897 & 117 & 1936 & 112 & 1975 & 121 \\
\hline 1820 & -- & 1859 & -- & 1898 & 108 & 1937 & 121 & 1976 & 109 \\
\hline 1821 & -- & 1860 & -- & 1899 & 119 & 1938 & 110 & 1977 & 109 \\
\hline 1822 & -- & 1861 & -- & 1900 & 119 & 1939 & 127 & 1978 & 123 \\
\hline 1823 & -- & 1862 & -- & 1901 & 111 & 1940 & 128 & 1979 & 116 \\
\hline 1824 & -- & 1863 & -- & 1902 & 106 & 1941 & 125 & 1980 & 104 \\
\hline 1825 & -- & 1864 & -- & 1903 & 101 & 1942 & 111 & 1981 & 91 \\
\hline 1826 & -- & 1865 & -- & 1904 & 124 & 1943 & 118 & 1982 & 120 \\
\hline 1827 & -- & 1866 & -- & 1905 & 112 & 1944 & 123 & 1983 & 99 \\
\hline 1828 & -- & 1867 & -- & 1906 & 116 & 1945 & 99 & 1984 & 112 \\
\hline 1829 & -- & 1868 & -- & 1907 & 120 & 1946 & 100 & 1985 & 102 \\
\hline 1830 & -- & 1869 & -- & 1908 & 117 & 1947 & 113 & 1986 & 107 \\
\hline 1831 & -- & 1870 & -- & 1909 & 117 & 1948 & 101 & 1987 & 107 \\
\hline 1832 & -- & 1871 & -- & 1910 & 96 & 1949 & 101 & 1988 & 101 \\
\hline 1833 & -- & 1872 & -- & 1911 & 119 & 1950 & 117 & 1989 & 116 \\
\hline 1834 & -- & 1873 & -- & 1912 & 116 & 1951 & 105 & 1990 & 101 \\
\hline 1835 & -- & 1874 & 133 & 1913 & 113 & 1952 & 111 & 1991 & 101 \\
\hline 1836 & -- & 1875 & 126 & 1914 & 116 & 1953 & 100 & 1992 & 113 \\
\hline 1837 & -- & 1876 & 132 & 1915 & 105 & 1954 & 113 & 1993 & 112 \\
\hline 1838 & -- & 1877 & 112 & 1916 & 112 & 1955 & 110 & 1994 & 116 \\
\hline 1839 & -- & 1878 & 102 & 1917 & 118 & 1956 & 123 & 1995 & 102 \\
\hline 1840 & -- & 1879 & 127 & 1918 & 114 & 1957 & 107 & 1996 & -- \\
\hline 1841 & -- & 1880 & 113 & 1919 & 104 & 1958 & 109 & 1997 & 117 \\
\hline 1842 & -- & 1881 & 119 & 1920 & 124 & 1959 & 114 & 1998 & 110 \\
\hline 1843 & -- & 1882 & 121 & 1921 & 98 & 1960 & 116 & 1999 & 113 \\
\hline 1844 & -- & 1883 & 122 & 1922 & 109 & 1961 & 120 & 2000 & -- \\
\hline 1845 & -- & 1884 & 117 & 1923 & 119 & 1962 & 113 & & \\
\hline
\end{tabular}


Table 16. Ice-out dates for Ponkapoag Pond, Massachusetts

$[--$, no data $]$

Location of Lake.-Lat. $42^{\circ} 11^{\prime} 31 "$, long. $71^{\circ} 05^{\prime} 40^{\prime \prime}$.

Period of Record.-1886 to 2000.

Years of Record.-115.

Observer.-Blue Hills Observatory.

Earliest Ice-Out Date.-1953: 32.

Latest Ice-Out Date.-1926: 100.

\begin{tabular}{|c|c|c|c|c|c|c|c|c|c|}
\hline Year & $\begin{array}{c}\text { Julian } \\
\text { day }\end{array}$ & Year & $\begin{array}{c}\text { Julian } \\
\text { day }\end{array}$ & Year & $\begin{array}{c}\text { Julian } \\
\text { day }\end{array}$ & Year & $\begin{array}{c}\text { Julian } \\
\text { day }\end{array}$ & Year & $\begin{array}{c}\text { Julian } \\
\text { day }\end{array}$ \\
\hline 1807 & -- & 1846 & -- & 1885 & -- & 1924 & 96 & 1963 & 86 \\
\hline 1808 & -- & 1847 & -- & 1886 & 86 & 1925 & 69 & 1964 & 77 \\
\hline 1809 & -- & 1848 & -- & 1887 & 83 & 1926 & 100 & 1965 & 72 \\
\hline 1810 & -- & 1849 & -- & 1888 & 89 & 1927 & 76 & 1966 & 80 \\
\hline 1811 & -- & 1850 & -- & 1889 & 71 & 1928 & 85 & 1967 & 94 \\
\hline 1812 & -- & 1851 & -- & 1890 & 71 & 1929 & 79 & 1968 & 86 \\
\hline 1813 & -- & 1852 & -- & 1891 & 72 & 1930 & 67 & 1969 & 93 \\
\hline 1814 & -- & 1853 & -- & 1892 & 93 & 1931 & 84 & 1970 & 76 \\
\hline 1815 & -- & 1854 & -- & 1893 & 91 & 1932 & 89 & 1971 & 87 \\
\hline 1816 & -- & 1855 & -- & 1894 & 71 & 1933 & 82 & 1972 & 83 \\
\hline 1817 & -- & 1856 & -- & 1895 & 86 & 1934 & 88 & 1973 & 66 \\
\hline 1818 & -- & 1857 & -- & 1896 & 93 & 1935 & 83 & 1974 & 63 \\
\hline 1819 & -- & 1858 & -- & 1897 & 82 & 1936 & 80 & 1975 & 75 \\
\hline 1820 & -- & 1859 & -- & 1898 & 73 & 1937 & 77 & 1976 & 60 \\
\hline 1821 & -- & 1860 & -- & 1899 & 90 & 1938 & 77 & 1977 & 75 \\
\hline 1822 & -- & 1861 & -- & 1900 & 79 & 1939 & 88 & 1978 & 92 \\
\hline 1823 & -- & 1862 & -- & 1901 & 82 & 1940 & 97 & 1979 & 82 \\
\hline 1824 & -- & 1863 & -- & 1902 & 73 & 1941 & 92 & 1980 & 71 \\
\hline 1825 & -- & 1864 & -- & 1903 & 70 & 1942 & 68 & 1981 & 52 \\
\hline 1826 & -- & 1865 & -- & 1904 & 94 & 1943 & 80 & 1982 & 74 \\
\hline 1827 & -- & 1866 & -- & 1905 & 90 & 1944 & 86 & 1983 & 67 \\
\hline 1828 & -- & 1867 & -- & 1906 & 90 & 1945 & 79 & 1984 & 52 \\
\hline 1829 & -- & 1868 & -- & 1907 & 86 & 1946 & 68 & 1985 & 59 \\
\hline 1830 & -- & 1869 & -- & 1908 & 77 & 1947 & 73 & 1986 & 66 \\
\hline 1831 & -- & 1870 & -- & 1909 & 76 & 1948 & 84 & 1987 & 89 \\
\hline 1832 & -- & 1871 & -- & 1910 & 81 & 1949 & 59 & 1988 & 70 \\
\hline 1833 & -- & 1872 & -- & 1911 & 87 & 1950 & 83 & 1989 & 54 \\
\hline 1834 & -- & 1873 & -- & 1912 & 82 & 1951 & 54 & 1990 & 41 \\
\hline 1835 & -- & 1874 & -- & 1913 & 73 & 1952 & 85 & 1991 & 52 \\
\hline 1836 & -- & 1875 & -- & 1914 & 90 & 1953 & 32 & 1992 & 58 \\
\hline 1837 & -- & 1876 & -- & 1915 & 71 & 1954 & 53 & 1993 & 94 \\
\hline 1838 & -- & 1877 & -- & 1916 & 96 & 1955 & 57 & 1994 & 93 \\
\hline 1839 & -- & 1878 & -- & 1917 & 92 & 1956 & 72 & 1995 & 68 \\
\hline 1840 & -- & 1879 & -- & 1918 & 91 & 1957 & 58 & 1996 & 57 \\
\hline 1841 & -- & 1880 & -- & 1919 & 65 & 1958 & 69 & 1997 & 51 \\
\hline 1842 & -- & 1881 & -- & 1920 & 94 & 1959 & 50 & 1998 & 43 \\
\hline 1843 & -- & 1882 & -- & 1921 & 69 & 1960 & 54 & 1999 & 36 \\
\hline 1844 & -- & 1883 & -- & 1922 & 85 & 1961 & 88 & 2000 & 63 \\
\hline 1845 & -- & 1884 & -- & 1923 & 99 & 1962 & 84 & & \\
\hline
\end{tabular}


Table 17. Ice-out dates for Pontoosuc Lake, Massachusetts

$[--$, no data $]$

Location of Lake.-Lat. $42^{\circ} 29^{\prime} 44^{\prime \prime}$, long. $73^{\circ} 14^{\prime} 59^{\prime \prime}$.

Period of Record.-1925 to 1998.

Years of Record. - 74 .

Observer.-Berkshire Eagle.

Earliest Ice-Out Date.-1995: 85.

Latest Ice-Out Date.-1940: 121.

\begin{tabular}{|c|c|c|c|c|c|c|c|c|c|}
\hline Year & $\begin{array}{c}\text { Julian } \\
\text { day }\end{array}$ & Year & $\begin{array}{c}\text { Julian } \\
\text { day }\end{array}$ & Year & $\begin{array}{c}\text { Julian } \\
\text { day }\end{array}$ & Year & $\begin{array}{c}\text { Julian } \\
\text { day }\end{array}$ & Year & $\begin{array}{c}\text { Julian } \\
\text { day }\end{array}$ \\
\hline 1807 & -- & 1846 & -- & 1885 & -- & 1924 & -- & 1963 & 106 \\
\hline 1808 & -- & 1847 & -- & 1886 & -- & 1925 & 92 & 1964 & 108 \\
\hline 1809 & -- & 1848 & -- & 1887 & -- & 1926 & 114 & 1965 & 108 \\
\hline 1810 & -- & 1849 & -- & 1888 & -- & 1927 & 98 & 1966 & 109 \\
\hline 1811 & -- & 1850 & -- & 1889 & -- & 1928 & 98 & 1967 & 110 \\
\hline 1812 & -- & 1851 & -- & 1890 & -- & 1929 & 97 & 1968 & 97 \\
\hline 1813 & -- & 1852 & -- & 1891 & -- & 1930 & 93 & 1969 & 106 \\
\hline 1814 & -- & 1853 & -- & 1892 & -- & 1931 & 100 & 1970 & 114 \\
\hline 1815 & -- & 1854 & -- & 1893 & -- & 1932 & 112 & 1971 & 115 \\
\hline 1816 & -- & 1855 & -- & 1894 & -- & 1933 & 107 & 1972 & 118 \\
\hline 1817 & -- & 1856 & -- & 1895 & -- & 1934 & 108 & 1973 & 90 \\
\hline 1818 & -- & 1857 & -- & 1896 & -- & 1935 & 100 & 1974 & 95 \\
\hline 1819 & -- & 1858 & -- & 1897 & -- & 1936 & 92 & 1975 & 112 \\
\hline 1820 & -- & 1859 & -- & 1898 & -- & 1937 & 105 & 1976 & 94 \\
\hline 1821 & -- & 1860 & -- & 1899 & -- & 1938 & 87 & 1977 & 104 \\
\hline 1822 & -- & 1861 & -- & 1900 & -- & 1939 & 113 & 1978 & 109 \\
\hline 1823 & -- & 1862 & -- & 1901 & -- & 1940 & 121 & 1979 & 111 \\
\hline 1824 & -- & 1863 & -- & 1902 & -- & 1941 & 105 & 1980 & 98 \\
\hline 1825 & -- & 1864 & -- & 1903 & -- & 1942 & 98 & 1981 & 92 \\
\hline 1826 & -- & 1865 & -- & 1904 & -- & 1943 & 116 & 1982 & 112 \\
\hline 1827 & -- & 1866 & -- & 1905 & -- & 1944 & 110 & 1983 & 92 \\
\hline 1828 & -- & 1867 & -- & 1906 & -- & 1945 & 89 & 1984 & 105 \\
\hline 1829 & -- & 1868 & -- & 1907 & -- & 1946 & 86 & 1985 & 92 \\
\hline 1830 & -- & 1869 & -- & 1908 & -- & 1947 & 104 & 1986 & 94 \\
\hline 1831 & -- & 1870 & -- & 1909 & -- & 1948 & 92 & 1987 & 95 \\
\hline 1832 & -- & 1871 & -- & 1910 & -- & 1949 & 87 & 1988 & 100 \\
\hline 1833 & -- & 1872 & -- & 1911 & -- & 1950 & 99 & 1989 & 88 \\
\hline 1834 & -- & 1873 & -- & 1912 & -- & 1951 & 100 & 1990 & 93 \\
\hline 1835 & -- & 1874 & -- & 1913 & -- & 1952 & 102 & 1991 & 86 \\
\hline 1836 & -- & 1875 & -- & 1914 & -- & 1953 & 86 & 1992 & 102 \\
\hline 1837 & -- & 1876 & -- & 1915 & -- & 1954 & 87 & 1993 & 107 \\
\hline 1838 & -- & 1877 & -- & 1916 & -- & 1955 & 105 & 1994 & 108 \\
\hline 1839 & -- & 1878 & -- & 1917 & -- & 1956 & 120 & 1995 & 85 \\
\hline 1840 & -- & 1879 & -- & 1918 & -- & 1957 & 90 & 1996 & 102 \\
\hline 1841 & -- & 1880 & -- & 1919 & -- & 1958 & 105 & 1997 & 99 \\
\hline 1842 & -- & 1881 & -- & 1920 & -- & 1959 & 108 & 1998 & 90 \\
\hline 1843 & -- & 1882 & -- & 1921 & -- & 1960 & 107 & 1999 & -- \\
\hline 1844 & -- & 1883 & -- & 1922 & -- & 1961 & 111 & 2000 & -- \\
\hline 1845 & -- & 1884 & -- & 1923 & -- & 1962 & 102 & & \\
\hline
\end{tabular}


Table 18. Ice-out dates for Portage Lake, Maine

[--, no data]

Location of Lake.-Lat. $46^{\circ} 46^{\prime} 17^{\prime \prime}$, long. $68^{\circ} 30^{\prime} 04^{\prime \prime}$.

Period of Record.-1925 to 2000.

Years of Record.-75.

Observer.-Portage Lake Town Office.

Earliest Ice-Out Date.-1945: 104.

Latest Ice-Out Date.—1926: 146.

\begin{tabular}{|c|c|c|c|c|c|c|c|c|c|}
\hline Year & $\begin{array}{c}\text { Julian } \\
\text { day }\end{array}$ & Year & $\begin{array}{c}\text { Julian } \\
\text { day }\end{array}$ & Year & $\begin{array}{c}\text { Julian } \\
\text { day }\end{array}$ & Year & $\begin{array}{c}\text { Julian } \\
\text { day }\end{array}$ & Year & $\begin{array}{c}\text { Julian } \\
\text { day }\end{array}$ \\
\hline 1807 & -- & 1846 & -- & 1885 & -- & 1924 & -- & 1963 & 131 \\
\hline 1808 & -- & 1847 & -- & 1886 & -- & 1925 & 130 & 1964 & 127 \\
\hline 1809 & -- & 1848 & -- & 1887 & -- & 1926 & 146 & 1965 & 124 \\
\hline 1810 & -- & 1849 & -- & 1888 & -- & 1927 & 120 & 1966 & 129 \\
\hline 1811 & -- & 1850 & -- & 1889 & -- & 1928 & 137 & 1967 & 127 \\
\hline 1812 & -- & 1851 & -- & 1890 & -- & 1929 & 130 & 1968 & 123 \\
\hline 1813 & -- & 1852 & -- & 1891 & -- & 1930 & 127 & 1969 & 131 \\
\hline 1814 & -- & 1853 & -- & 1892 & -- & 1931 & 121 & 1970 & 133 \\
\hline 1815 & -- & 1854 & -- & 1893 & -- & 1932 & 129 & 1971 & 133 \\
\hline 1816 & -- & 1855 & -- & 1894 & -- & 1933 & 127 & 1972 & 140 \\
\hline 1817 & -- & 1856 & -- & 1895 & -- & 1934 & 120 & 1973 & 127 \\
\hline 1818 & -- & 1857 & -- & 1896 & -- & 1935 & 132 & 1974 & 141 \\
\hline 1819 & -- & 1858 & -- & 1897 & -- & 1936 & 124 & 1975 & 135 \\
\hline 1820 & -- & 1859 & -- & 1898 & -- & 1937 & 133 & 1976 & 123 \\
\hline 1821 & -- & 1860 & -- & 1899 & -- & 1938 & 118 & 1977 & 132 \\
\hline 1822 & -- & 1861 & -- & 1900 & -- & 1939 & 133 & 1978 & 132 \\
\hline 1823 & -- & 1862 & -- & 1901 & -- & 1940 & 132 & 1979 & 123 \\
\hline 1824 & -- & 1863 & -- & 1902 & -- & 1941 & 122 & 1980 & 125 \\
\hline 1825 & -- & 1864 & -- & 1903 & -- & 1942 & 124 & 1981 & 121 \\
\hline 1826 & -- & 1865 & -- & 1904 & -- & 1943 & 139 & 1982 & 129 \\
\hline 1827 & -- & 1866 & -- & 1905 & -- & 1944 & 129 & 1983 & 121 \\
\hline 1828 & -- & 1867 & -- & 1906 & -- & 1945 & 104 & 1984 & 129 \\
\hline 1829 & -- & 1868 & -- & 1907 & -- & 1946 & 131 & 1985 & 126 \\
\hline 1830 & -- & 1869 & -- & 1908 & -- & 1947 & 138 & 1986 & 118 \\
\hline 1831 & -- & 1870 & -- & 1909 & -- & 1948 & 126 & 1987 & 110 \\
\hline 1832 & -- & 1871 & -- & 1910 & -- & 1949 & 124 & 1988 & 119 \\
\hline 1833 & -- & 1872 & -- & 1911 & -- & 1950 & 127 & 1989 & 125 \\
\hline 1834 & -- & 1873 & -- & 1912 & -- & 1951 & 119 & 1990 & 120 \\
\hline 1835 & -- & 1874 & -- & 1913 & -- & 1952 & 129 & 1991 & 125 \\
\hline 1836 & -- & 1875 & -- & 1914 & -- & 1953 & 125 & 1992 & 132 \\
\hline 1837 & -- & 1876 & -- & 1915 & -- & 1954 & 122 & 1993 & 123 \\
\hline 1838 & -- & 1877 & -- & 1916 & -- & 1955 & 123 & 1994 & 132 \\
\hline 1839 & -- & 1878 & -- & 1917 & -- & 1956 & 133 & 1995 & 124 \\
\hline 1840 & -- & 1879 & -- & 1918 & -- & 1957 & 122 & 1996 & 124 \\
\hline 1841 & -- & 1880 & -- & 1919 & -- & 1958 & -- & 1997 & 126 \\
\hline 1842 & -- & 1881 & -- & 1920 & -- & 1959 & 129 & 1998 & 120 \\
\hline 1843 & -- & 1882 & -- & 1921 & -- & 1960 & 127 & 1999 & 123 \\
\hline 1844 & -- & 1883 & -- & 1922 & -- & 1961 & 135 & 2000 & 121 \\
\hline 1845 & -- & 1884 & -- & 1923 & -- & 1962 & 130 & & \\
\hline
\end{tabular}


Table 19. Ice-out dates for Rangeley Lake, Maine

[--, no data]

Location of Lake.-Lat. $44^{\circ} 56^{\prime} 41^{\prime \prime}$, long. 70 41'54".

Period of Record.-1880 to 2000.

Years of Record. -121 .

Observers.-Union Water Power Company, FPL Energy Maine.

Earliest Ice-Out Date.-1921, 1945: 104.

Latest Ice-Out Date.-1882, 1972: 144.

\begin{tabular}{|c|c|c|c|c|c|c|c|c|c|}
\hline Year & $\begin{array}{c}\text { Julian } \\
\text { day }\end{array}$ & Year & $\begin{array}{c}\text { Julian } \\
\text { day }\end{array}$ & Year & $\begin{array}{c}\text { Julian } \\
\text { day }\end{array}$ & Year & $\begin{array}{c}\text { Julian } \\
\text { day }\end{array}$ & Year & $\begin{array}{c}\text { Julian } \\
\text { day }\end{array}$ \\
\hline 1807 & -- & 1846 & -- & 1885 & 136 & 1924 & 131 & 1963 & 127 \\
\hline 1808 & -- & 1847 & -- & 1886 & 124 & 1925 & 119 & 1964 & 127 \\
\hline 1809 & -- & 1848 & -- & 1887 & 137 & 1926 & 138 & 1965 & 129 \\
\hline 1810 & -- & 1849 & -- & 1888 & 142 & 1927 & 119 & 1966 & 137 \\
\hline 1811 & -- & 1850 & -- & 1889 & 120 & 1928 & 134 & 1967 & 130 \\
\hline 1812 & -- & 1851 & -- & 1890 & 129 & 1929 & 130 & 1968 & 116 \\
\hline 1813 & -- & 1852 & -- & 1891 & 134 & 1930 & 126 & 1969 & 132 \\
\hline 1814 & -- & 1853 & -- & 1892 & 130 & 1931 & 116 & 1970 & 135 \\
\hline 1815 & -- & 1854 & -- & 1893 & 137 & 1932 & 127 & 1971 & 135 \\
\hline 1816 & -- & 1855 & -- & 1894 & 122 & 1933 & 127 & 1972 & 144 \\
\hline 1817 & -- & 1856 & -- & 1895 & 127 & 1934 & 125 & 1973 & 124 \\
\hline 1818 & -- & 1857 & -- & 1896 & 131 & 1935 & 131 & 1974 & 135 \\
\hline 1819 & -- & 1858 & -- & 1897 & 133 & 1936 & 125 & 1975 & 132 \\
\hline 1820 & -- & 1859 & -- & 1898 & 125 & 1937 & 131 & 1976 & 119 \\
\hline 1821 & -- & 1860 & -- & 1899 & 128 & 1938 & 119 & 1977 & 127 \\
\hline 1822 & -- & 1861 & -- & 1900 & 136 & 1939 & 134 & 1978 & 137 \\
\hline 1823 & -- & 1862 & -- & 1901 & 124 & 1940 & 140 & 1979 & 121 \\
\hline 1824 & -- & 1863 & -- & 1902 & 118 & 1941 & 116 & 1980 & 126 \\
\hline 1825 & -- & 1864 & -- & 1903 & 117 & 1942 & 125 & 1981 & 120 \\
\hline 1826 & -- & 1865 & -- & 1904 & 135 & 1943 & 140 & 1982 & 133 \\
\hline 1827 & -- & 1866 & -- & 1905 & 125 & 1944 & 132 & 1983 & 126 \\
\hline 1828 & -- & 1867 & -- & 1906 & 133 & 1945 & 104 & 1984 & 125 \\
\hline 1829 & -- & 1868 & -- & 1907 & 139 & 1946 & 122 & 1985 & 130 \\
\hline 1830 & -- & 1869 & -- & 1908 & 131 & 1947 & 133 & 1986 & 117 \\
\hline 1831 & -- & 1870 & -- & 1909 & 132 & 1948 & 122 & 1987 & 111 \\
\hline 1832 & -- & 1871 & -- & 1910 & 109 & 1949 & 114 & 1988 & 126 \\
\hline 1833 & -- & 1872 & -- & 1911 & 131 & 1950 & 128 & 1989 & 131 \\
\hline 1834 & -- & 1873 & -- & 1912 & 130 & 1951 & 122 & 1990 & 125 \\
\hline 1835 & -- & 1874 & -- & 1913 & 124 & 1952 & 123 & 1991 & 121 \\
\hline 1836 & -- & 1875 & -- & 1914 & 141 & 1953 & 114 & 1992 & 133 \\
\hline 1837 & -- & 1876 & -- & 1915 & 119 & 1954 & 126 & 1993 & 122 \\
\hline 1838 & -- & 1877 & -- & 1916 & 129 & 1955 & 120 & 1994 & 128 \\
\hline 1839 & -- & 1878 & -- & 1917 & 137 & 1956 & 136 & 1995 & 125 \\
\hline 1840 & -- & 1879 & -- & 1918 & 123 & 1957 & 121 & 1996 & 130 \\
\hline 1841 & -- & 1880 & 134 & 1919 & 123 & 1958 & 127 & 1997 & 133 \\
\hline 1842 & -- & 1881 & 136 & 1920 & 140 & 1959 & 128 & 1998 & 113 \\
\hline 1843 & -- & 1882 & 144 & 1921 & 104 & 1960 & 125 & 1999 & 123 \\
\hline 1844 & -- & 1883 & 136 & 1922 & 124 & 1961 & 135 & 2000 & 121 \\
\hline 1845 & -- & 1884 & 134 & 1923 & 129 & 1962 & 137 & & \\
\hline
\end{tabular}


Table 20. Ice-out dates for Richardson Lake, Maine

[--, no data]

Location of Lake.-Lat. $44^{\circ} 51^{\prime} 23 "$, long. $70^{\circ} 51^{\prime} 56^{\prime \prime}$.

Period of Record.-1880 to 2000.

Years of Record.-119.

Observers.-Union Water Power Company, FPL Energy Maine.

Earliest Ice-Out Date.-1945: 104.

Latest Ice-Out Date.-1888: 144.

\begin{tabular}{|c|c|c|c|c|c|c|c|c|c|}
\hline Year & $\begin{array}{c}\text { Julian } \\
\text { day }\end{array}$ & Year & $\begin{array}{c}\text { Julian } \\
\text { day }\end{array}$ & Year & $\begin{array}{c}\text { Julian } \\
\text { day }\end{array}$ & Year & $\begin{array}{c}\text { Julian } \\
\text { day }\end{array}$ & Year & $\begin{array}{c}\text { Julian } \\
\text { day }\end{array}$ \\
\hline 1807 & -- & 1846 & -- & 1885 & 133 & 1924 & 129 & 1963 & 125 \\
\hline 1808 & -- & 1847 & -- & 1886 & 122 & 1925 & 118 & 1964 & 127 \\
\hline 1809 & -- & 1848 & -- & 1887 & 132 & 1926 & 135 & 1965 & 127 \\
\hline 1810 & -- & 1849 & -- & 1888 & 144 & 1927 & 112 & 1966 & 129 \\
\hline 1811 & -- & 1850 & -- & 1889 & 119 & 1928 & 132 & 1967 & 129 \\
\hline 1812 & -- & 1851 & -- & 1890 & 131 & 1929 & 129 & 1968 & 114 \\
\hline 1813 & -- & 1852 & -- & 1891 & 126 & 1930 & 125 & 1969 & 130 \\
\hline 1814 & -- & 1853 & -- & 1892 & 127 & 1931 & 114 & 1970 & 134 \\
\hline 1815 & -- & 1854 & -- & 1893 & 141 & 1932 & 125 & 1971 & 132 \\
\hline 1816 & -- & 1855 & -- & 1894 & 121 & 1933 & 127 & 1972 & 141 \\
\hline 1817 & -- & 1856 & -- & 1895 & 125 & 1934 & 124 & 1973 & 124 \\
\hline 1818 & -- & 1857 & -- & 1896 & 130 & 1935 & 131 & 1974 & 133 \\
\hline 1819 & -- & 1858 & -- & 1897 & 132 & 1936 & 124 & 1975 & 130 \\
\hline 1820 & -- & 1859 & -- & 1898 & 121 & 1937 & 133 & 1976 & 116 \\
\hline 1821 & -- & 1860 & -- & 1899 & 128 & 1938 & -- & 1977 & 125 \\
\hline 1822 & -- & 1861 & -- & 1900 & 130 & 1939 & 134 & 1978 & 136 \\
\hline 1823 & -- & 1862 & -- & 1901 & 121 & 1940 & -- & 1979 & 119 \\
\hline 1824 & -- & 1863 & -- & 1902 & 113 & 1941 & 116 & 1980 & 124 \\
\hline 1825 & -- & 1864 & -- & 1903 & 117 & 1942 & 123 & 1981 & 117 \\
\hline 1826 & -- & 1865 & -- & 1904 & 130 & 1943 & 139 & 1982 & 131 \\
\hline 1827 & -- & 1866 & -- & 1905 & 123 & 1944 & 130 & 1983 & 126 \\
\hline 1828 & -- & 1867 & -- & 1906 & 136 & 1945 & 104 & 1984 & 123 \\
\hline 1829 & -- & 1868 & -- & 1907 & 137 & 1946 & 122 & 1985 & 127 \\
\hline 1830 & -- & 1869 & -- & 1908 & 128 & 1947 & 133 & 1986 & 115 \\
\hline 1831 & -- & 1870 & -- & 1909 & 131 & 1948 & 117 & 1987 & 110 \\
\hline 1832 & -- & 1871 & -- & 1910 & 107 & 1949 & 117 & 1988 & 120 \\
\hline 1833 & -- & 1872 & -- & 1911 & 130 & 1950 & 127 & 1989 & 131 \\
\hline 1834 & -- & 1873 & -- & 1912 & 128 & 1951 & 123 & 1990 & 125 \\
\hline 1835 & -- & 1874 & -- & 1913 & 123 & 1952 & 121 & 1991 & 121 \\
\hline 1836 & -- & 1875 & -- & 1914 & 136 & 1953 & 112 & 1992 & 134 \\
\hline 1837 & -- & 1876 & -- & 1915 & 118 & 1954 & 122 & 1993 & 122 \\
\hline 1838 & -- & 1877 & -- & 1916 & 129 & 1955 & 119 & 1994 & 129 \\
\hline 1839 & -- & 1878 & -- & 1917 & 137 & 1956 & 135 & 1995 & 126 \\
\hline 1840 & -- & 1879 & -- & 1918 & 120 & 1957 & 121 & 1996 & 129 \\
\hline 1841 & -- & 1880 & 129 & 1919 & 123 & 1958 & 122 & 1997 & 128 \\
\hline 1842 & -- & 1881 & 135 & 1920 & 138 & 1959 & 125 & 1998 & 109 \\
\hline 1843 & -- & 1882 & 141 & 1921 & 105 & 1960 & 122 & 1999 & 122 \\
\hline 1844 & -- & 1883 & 134 & 1922 & 124 & 1961 & 135 & 2000 & 121 \\
\hline 1845 & -- & 1884 & 133 & 1923 & 127 & 1962 & 129 & & \\
\hline
\end{tabular}


Table 21. Ice-out dates for Sebago Lake, Maine

[--, no data; NCIC, no complete ice cover]

Location of Lake.-Lat. $43^{\circ} 52^{\prime} 15^{\prime \prime}$, long. $70^{\circ} 34^{\prime} 01^{\prime \prime}$.

Period of Record.-1807 to 2000.

Years of Record.- 160 .

Observers and Ice-Out Definition.--Maine Sunday Telegram, Carroll Cutting, Portland Press Herald. Ice-out thought to be observed on Big Bay for many years.

Earliest Ice-Out Date.—1937, 1947, 1949, 1951, 1953, 1961, 1991, 1998, 1999: No complete ice cover.

Latest Ice-Out Date.-1888: 129.

\begin{tabular}{|c|c|c|c|c|c|c|c|c|c|}
\hline Year & $\begin{array}{c}\text { Julian } \\
\text { day }\end{array}$ & Year & $\begin{array}{c}\text { Julian } \\
\text { day }\end{array}$ & Year & $\begin{array}{c}\text { Julian } \\
\text { day }\end{array}$ & Year & $\begin{array}{c}\text { Julian } \\
\text { day }\end{array}$ & Year & $\begin{array}{c}\text { Julian } \\
\text { day }\end{array}$ \\
\hline 1807 & 127 & 1846 & 104 & 1885 & 116 & 1924 & 107 & 1963 & 105 \\
\hline 1808 & -- & 1847 & -- & 1886 & 115 & 1925 & 94 & 1964 & 107 \\
\hline 1809 & -- & 1848 & -- & 1887 & 121 & 1926 & 120 & 1965 & 104 \\
\hline 1810 & -- & 1849 & 119 & 1888 & 129 & 1927 & 105 & 1966 & 92 \\
\hline 1811 & -- & 1850 & -- & 1889 & 102 & 1928 & 99 & 1967 & 100 \\
\hline 1812 & 122 & 1851 & 110 & 1890 & 114 & 1929 & 100 & 1968 & 99 \\
\hline 1813 & -- & 1852 & 125 & 1891 & 113 & 1930 & 97 & 1969 & 111 \\
\hline 1814 & -- & 1853 & 110 & 1892 & 102 & 1931 & 102 & 1970 & 113 \\
\hline 1815 & -- & 1854 & -- & 1893 & 124 & 1932 & 73 & 1971 & 122 \\
\hline 1816 & 121 & 1855 & 117 & 1894 & 109 & 1933 & 100 & 1972 & 122 \\
\hline 1817 & -- & 1856 & 118 & 1895 & 111 & 1934 & 110 & 1973 & 100 \\
\hline 1818 & -- & 1857 & 104 & 1896 & 112 & 1935 & 107 & 1974 & 101 \\
\hline 1819 & 119 & 1858 & 106 & 1897 & 112 & 1936 & 87 & 1975 & 88 \\
\hline 1820 & 116 & 1859 & -- & 1898 & 103 & 1937 & NCIC & 1976 & 94 \\
\hline 1821 & 119 & 1860 & -- & 1899 & 117 & 1938 & 92 & 1977 & 94 \\
\hline 1822 & 102 & 1861 & -- & 1900 & 116 & 1939 & 113 & 1978 & 104 \\
\hline 1823 & 113 & 1862 & 119 & 1901 & 105 & 1940 & 124 & 1979 & 85 \\
\hline 1824 & 108 & 1863 & 118 & 1902 & 89 & 1941 & 102 & 1980 & 102 \\
\hline 1825 & 106 & 1864 & -- & 1903 & 87 & 1942 & 98 & 1981 & 76 \\
\hline 1826 & 108 & 1865 & -- & 1904 & 114 & 1943 & 107 & 1982 & 111 \\
\hline 1827 & -- & 1866 & 108 & 1905 & 115 & 1944 & 115 & 1983 & 76 \\
\hline 1828 & -- & 1867 & 116 & 1906 & 112 & 1945 & 91 & 1984 & 107 \\
\hline 1829 & -- & 1868 & -- & 1907 & 120 & 1946 & 85 & 1985 & 73 \\
\hline 1830 & -- & 1869 & -- & 1908 & 116 & 1947 & NCIC & 1986 & 90 \\
\hline 1831 & -- & 1870 & -- & 1909 & 105 & 1948 & 94 & 1987 & 88 \\
\hline 1832 & -- & 1871 & 95 & 1910 & 93 & 1949 & NCIC & 1988 & 92 \\
\hline 1833 & -- & 1872 & 125 & 1911 & 119 & 1950 & 107 & 1989 & 100 \\
\hline 1834 & 104 & 1873 & 121 & 1912 & 114 & 1951 & NCIC & 1990 & 95 \\
\hline 1835 & -- & 1874 & 127 & 1913 & 99 & 1952 & 106 & 1991 & NCIC \\
\hline 1836 & -- & 1875 & 126 & 1914 & 110 & 1953 & NCIC & 1992 & 87 \\
\hline 1837 & 121 & 1876 & 122 & 1915 & 102 & 1954 & 103 & 1993 & 109 \\
\hline 1838 & -- & 1877 & 113 & 1916 & 109 & 1955 & -- & 1994 & 110 \\
\hline 1839 & -- & 1878 & 102 & 1917 & 114 & 1956 & 97 & 1995 & 77 \\
\hline 1840 & 110 & 1879 & 125 & 1918 & 116 & 1957 & 99 & 1996 & 95 \\
\hline 1841 & 119 & 1880 & 104 & 1919 & 91 & 1958 & 96 & 1997 & 104 \\
\hline 1842 & 110 & 1881 & 114 & 1920 & 114 & 1959 & 96 & 1998 & NCIC \\
\hline 1843 & 122 & 1882 & 109 & 1921 & 87 & 1960 & -- & 1999 & NCIC \\
\hline 1844 & 108 & 1883 & 119 & 1922 & 104 & 1961 & NCIC & 2000 & 89 \\
\hline 1845 & 114 & 1884 & 117 & 1923 & 112 & 1962 & 100 & & \\
\hline
\end{tabular}


Table 22. Ice-out dates for Sebec Lake, Maine

[--, no data]

Location of Lake.-Lat. $45^{\circ} 16^{\prime} 58^{\prime \prime}$, long. $69^{\circ} 16^{\prime} 45^{\prime \prime}$.

Period of Record.- -1879 to 2000.

Years of Record.-120.

Observers and Ice-Out Definition.-Bill Elliot, Bill Larabee, Ruth Weatherbee. Ice-out definition for most years thought be date of open navigation from one end of lake to the other.

Earliest Ice-Out Date.-1902: 91.

Latest Ice-Out Date.-1972: 135.

\begin{tabular}{|c|c|c|c|c|c|c|c|c|c|}
\hline Year & $\begin{array}{c}\text { Julian } \\
\text { day }\end{array}$ & Year & $\begin{array}{c}\text { Julian } \\
\text { day }\end{array}$ & Year & $\begin{array}{c}\text { Julian } \\
\text { day }\end{array}$ & Year & $\begin{array}{c}\text { Julian } \\
\text { day }\end{array}$ & Year & $\begin{array}{c}\text { Julian } \\
\text { day }\end{array}$ \\
\hline 1807 & -- & 1846 & -- & 1885 & 131 & 1924 & 120 & 1963 & 118 \\
\hline 1808 & -- & 1847 & -- & 1886 & 122 & 1925 & 113 & 1964 & 122 \\
\hline 1809 & -- & 1848 & -- & 1887 & 118 & 1926 & 130 & 1965 & 121 \\
\hline 1810 & -- & 1849 & -- & 1888 & 133 & 1927 & 111 & 1966 & 116 \\
\hline 1811 & -- & 1850 & -- & 1889 & 114 & 1928 & 129 & 1967 & 124 \\
\hline 1812 & -- & 1851 & -- & 1890 & -- & 1929 & 123 & 1968 & 119 \\
\hline 1813 & -- & 1852 & -- & 1891 & 116 & 1930 & 121 & 1969 & 125 \\
\hline 1814 & -- & 1853 & -- & 1892 & 121 & 1931 & 104 & 1970 & 127 \\
\hline 1815 & -- & 1854 & -- & 1893 & 132 & 1932 & 118 & 1971 & 127 \\
\hline 1816 & -- & 1855 & -- & 1894 & 118 & 1933 & 121 & 1972 & 135 \\
\hline 1817 & -- & 1856 & -- & 1895 & 119 & 1934 & 116 & 1973 & 117 \\
\hline 1818 & -- & 1857 & -- & 1896 & 125 & 1935 & 118 & 1974 & 121 \\
\hline 1819 & -- & 1858 & -- & 1897 & 124 & 1936 & 119 & 1975 & 125 \\
\hline 1820 & -- & 1859 & -- & 1898 & 118 & 1937 & 125 & 1976 & 119 \\
\hline 1821 & -- & 1860 & -- & 1899 & 124 & 1938 & 117 & 1977 & 118 \\
\hline 1822 & -- & 1861 & -- & 1900 & 121 & 1939 & 131 & 1978 & 128 \\
\hline 1823 & -- & 1862 & -- & 1901 & 112 & 1940 & 131 & 1979 & 118 \\
\hline 1824 & -- & 1863 & -- & 1902 & 91 & 1941 & 112 & 1980 & 115 \\
\hline 1825 & -- & 1864 & -- & 1903 & 107 & 1942 & 119 & 1981 & 104 \\
\hline 1826 & -- & 1865 & -- & 1904 & -- & 1943 & 128 & 1982 & 126 \\
\hline 1827 & -- & 1866 & -- & 1905 & 119 & 1944 & 126 & 1983 & 110 \\
\hline 1828 & -- & 1867 & -- & 1906 & 128 & 1945 & 100 & 1984 & 119 \\
\hline 1829 & -- & 1868 & -- & 1907 & 125 & 1946 & 117 & 1985 & 117 \\
\hline 1830 & -- & 1869 & -- & 1908 & 126 & 1947 & 126 & 1986 & 114 \\
\hline 1831 & -- & 1870 & -- & 1909 & 129 & 1948 & 121 & 1987 & 103 \\
\hline 1832 & -- & 1871 & -- & 1910 & 110 & 1949 & 105 & 1988 & 115 \\
\hline 1833 & -- & 1872 & -- & 1911 & 126 & 1950 & 122 & 1989 & 123 \\
\hline 1834 & -- & 1873 & -- & 1912 & 125 & 1951 & 111 & 1990 & 121 \\
\hline 1835 & -- & 1874 & -- & 1913 & 117 & 1952 & 115 & 1991 & 120 \\
\hline 1836 & -- & 1875 & -- & 1914 & 129 & 1953 & 107 & 1992 & 128 \\
\hline 1837 & -- & 1876 & -- & 1915 & 115 & 1954 & 120 & 1993 & 122 \\
\hline 1838 & -- & 1877 & -- & 1916 & 116 & 1955 & 121 & 1994 & 122 \\
\hline 1839 & -- & 1878 & -- & 1917 & 127 & 1956 & 127 & 1995 & 114 \\
\hline 1840 & -- & 1879 & 128 & 1918 & 116 & 1957 & 117 & 1996 & 117 \\
\hline 1841 & -- & 1880 & 127 & 1919 & 114 & 1958 & 114 & 1997 & 122 \\
\hline 1842 & -- & 1881 & 118 & 1920 & 129 & 1959 & 118 & 1998 & 108 \\
\hline 1843 & -- & 1882 & 128 & 1921 & 107 & 1960 & 123 & 1999 & 108 \\
\hline 1844 & -- & 1883 & 124 & 1922 & 111 & 1961 & 123 & 2000 & 111 \\
\hline 1845 & -- & 1884 & 119 & 1923 & 126 & 1962 & 124 & & \\
\hline
\end{tabular}


Table 23. Ice-out dates for Squa Pan Lake, Maine

$[--$, no data $]$

Location of Lake.-Lat. $46^{\circ} 33^{\prime} 21^{\prime \prime}$, long. 68 $18^{\prime} 24^{\prime \prime}$.

Period of Record.-1930 to 1999.

Years of Record. - 64.

Observers. - Maine Public Service Company, WPS New England Generation.

Earliest Ice-Out Date.-1945: 103.

Latest Ice-Out Date. - 1972: 138.

\begin{tabular}{|c|c|c|c|c|c|c|c|c|c|}
\hline Year & $\begin{array}{c}\text { Julian } \\
\text { day }\end{array}$ & Year & $\begin{array}{c}\text { Julian } \\
\text { day }\end{array}$ & Year & $\begin{array}{c}\text { Julian } \\
\text { day }\end{array}$ & Year & $\begin{array}{c}\text { Julian } \\
\text { day }\end{array}$ & Year & $\begin{array}{c}\text { Julian } \\
\text { day }\end{array}$ \\
\hline 1807 & -- & 1846 & -- & 1885 & -- & 1924 & -- & 1963 & 126 \\
\hline 1808 & -- & 1847 & -- & 1886 & -- & 1925 & -- & 1964 & 123 \\
\hline 1809 & -- & 1848 & -- & 1887 & -- & 1926 & -- & 1965 & 122 \\
\hline 1810 & -- & 1849 & -- & 1888 & -- & 1927 & -- & 1966 & 117 \\
\hline 1811 & -- & 1850 & -- & 1889 & -- & 1928 & -- & 1967 & -- \\
\hline 1812 & -- & 1851 & -- & 1890 & -- & 1929 & -- & 1968 & 116 \\
\hline 1813 & -- & 1852 & -- & 1891 & -- & 1930 & 126 & 1969 & 125 \\
\hline 1814 & -- & 1853 & -- & 1892 & -- & 1931 & 112 & 1970 & 128 \\
\hline 1815 & -- & 1854 & -- & 1893 & -- & 1932 & -- & 1971 & 132 \\
\hline 1816 & -- & 1855 & -- & 1894 & -- & 1933 & 125 & 1972 & 138 \\
\hline 1817 & -- & 1856 & -- & 1895 & -- & 1934 & 118 & 1973 & 124 \\
\hline 1818 & -- & 1857 & -- & 1896 & -- & 1935 & -- & 1974 & 135 \\
\hline 1819 & -- & 1858 & -- & 1897 & -- & 1936 & -- & 1975 & 132 \\
\hline 1820 & -- & 1859 & -- & 1898 & -- & 1937 & 123 & 1976 & 120 \\
\hline 1821 & -- & 1860 & -- & 1899 & -- & 1938 & 116 & 1977 & 126 \\
\hline 1822 & -- & 1861 & -- & 1900 & -- & 1939 & 131 & 1978 & 129 \\
\hline 1823 & -- & 1862 & -- & 1901 & -- & 1940 & 128 & 1979 & 119 \\
\hline 1824 & -- & 1863 & -- & 1902 & -- & 1941 & 121 & 1980 & 123 \\
\hline 1825 & -- & 1864 & -- & 1903 & -- & 1942 & -- & 1981 & 119 \\
\hline 1826 & -- & 1865 & -- & 1904 & -- & 1943 & 136 & 1982 & 128 \\
\hline 1827 & -- & 1866 & -- & 1905 & -- & 1944 & -- & 1983 & 116 \\
\hline 1828 & -- & 1867 & -- & 1906 & -- & 1945 & 103 & 1984 & 122 \\
\hline 1829 & -- & 1868 & -- & 1907 & -- & 1946 & 126 & 1985 & 119 \\
\hline 1830 & -- & 1869 & -- & 1908 & -- & 1947 & 132 & 1986 & 113 \\
\hline 1831 & -- & 1870 & -- & 1909 & -- & 1948 & 108 & 1987 & 107 \\
\hline 1832 & -- & 1871 & -- & 1910 & -- & 1949 & 114 & 1988 & 117 \\
\hline 1833 & -- & 1872 & -- & 1911 & -- & 1950 & 125 & 1989 & 123 \\
\hline 1834 & -- & 1873 & -- & 1912 & -- & 1951 & 115 & 1990 & 121 \\
\hline 1835 & -- & 1874 & -- & 1913 & -- & 1952 & 122 & 1991 & 123 \\
\hline 1836 & -- & 1875 & -- & 1914 & -- & 1953 & 116 & 1992 & 130 \\
\hline 1837 & -- & 1876 & -- & 1915 & -- & 1954 & 119 & 1993 & 122 \\
\hline 1838 & -- & 1877 & -- & 1916 & -- & 1955 & 120 & 1994 & 127 \\
\hline 1839 & -- & 1878 & -- & 1917 & -- & 1956 & 127 & 1995 & 122 \\
\hline 1840 & -- & 1879 & -- & 1918 & -- & 1957 & 124 & 1996 & 120 \\
\hline 1841 & -- & 1880 & -- & 1919 & -- & 1958 & 119 & 1997 & 125 \\
\hline 1842 & -- & 1881 & -- & 1920 & -- & 1959 & 128 & 1998 & 118 \\
\hline 1843 & -- & 1882 & -- & 1921 & -- & 1960 & 129 & 1999 & 120 \\
\hline 1844 & -- & 1883 & -- & 1922 & -- & 1961 & 136 & 2000 & -- \\
\hline 1845 & -- & 1884 & -- & 1923 & -- & 1962 & 128 & & \\
\hline
\end{tabular}


Table 24. Ice-out dates for Sunapee Lake, New Hampshire

$[--$, no data]

Location of Lake.-Lat. $43^{\circ} 23^{\prime} 45^{\prime \prime}$, long. $72^{\circ} 03^{\prime} 11^{\prime \prime}$.

Period of Record.-1869 to 2000.

Years of Record.-132.

Observer.-Argus Champion.

Earliest Ice-Out Date.-1921, 1946: 88.

Latest Ice-Out Date.—1888: 135.

\begin{tabular}{|c|c|c|c|c|c|c|c|c|c|}
\hline Year & $\begin{array}{c}\text { Julian } \\
\text { day }\end{array}$ & Year & $\begin{array}{c}\text { Julian } \\
\text { day }\end{array}$ & Year & $\begin{array}{c}\text { Julian } \\
\text { day }\end{array}$ & Year & $\begin{array}{c}\text { Julian } \\
\text { day }\end{array}$ & Year & $\begin{array}{c}\text { Julian } \\
\text { day }\end{array}$ \\
\hline 1807 & -- & 1846 & -- & 1885 & 120 & 1924 & 110 & 1963 & 110 \\
\hline 1808 & -- & 1847 & -- & 1886 & 114 & 1925 & 116 & 1964 & 119 \\
\hline 1809 & -- & 1848 & -- & 1887 & 127 & 1926 & 124 & 1965 & 118 \\
\hline 1810 & -- & 1849 & -- & 1888 & 135 & 1927 & 110 & 1966 & 116 \\
\hline 1811 & -- & 1850 & -- & 1889 & 110 & 1928 & 121 & 1967 & 118 \\
\hline 1812 & -- & 1851 & -- & 1890 & 116 & 1929 & 104 & 1968 & 105 \\
\hline 1813 & -- & 1852 & -- & 1891 & 114 & 1930 & 107 & 1969 & 116 \\
\hline 1814 & -- & 1853 & -- & 1892 & 107 & 1931 & 106 & 1970 & 121 \\
\hline 1815 & -- & 1854 & -- & 1893 & 133 & 1932 & 117 & 1971 & 125 \\
\hline 1816 & -- & 1855 & -- & 1894 & 109 & 1933 & 120 & 1972 & 127 \\
\hline 1817 & -- & 1856 & -- & 1895 & 120 & 1934 & 114 & 1973 & 109 \\
\hline 1818 & -- & 1857 & -- & 1896 & 119 & 1935 & 115 & 1974 & 110 \\
\hline 1819 & -- & 1858 & -- & 1897 & 119 & 1936 & 105 & 1975 & 125 \\
\hline 1820 & -- & 1859 & -- & 1898 & 108 & 1937 & 122 & 1976 & 107 \\
\hline 1821 & -- & 1860 & -- & 1899 & 123 & 1938 & 108 & 1977 & 109 \\
\hline 1822 & -- & 1861 & -- & 1900 & 120 & 1939 & 127 & 1978 & 121 \\
\hline 1823 & -- & 1862 & -- & 1901 & 114 & 1940 & 128 & 1979 & 118 \\
\hline 1824 & -- & 1863 & -- & 1902 & 102 & 1941 & 108 & 1980 & 111 \\
\hline 1825 & -- & 1864 & -- & 1903 & 95 & 1942 & 109 & 1981 & 99 \\
\hline 1826 & -- & 1865 & -- & 1904 & 122 & 1943 & 123 & 1982 & 118 \\
\hline 1827 & -- & 1866 & -- & 1905 & 115 & 1944 & 122 & 1983 & 106 \\
\hline 1828 & -- & 1867 & -- & 1906 & 119 & 1945 & 91 & 1984 & 112 \\
\hline 1829 & -- & 1868 & -- & 1907 & 121 & 1946 & 88 & 1985 & 106 \\
\hline 1830 & -- & 1869 & 130 & 1908 & 117 & 1947 & 117 & 1986 & 105 \\
\hline 1831 & -- & 1870 & 130 & 1909 & 112 & 1948 & 99 & 1987 & 103 \\
\hline 1832 & -- & 1871 & 102 & 1910 & 96 & 1949 & 96 & 1988 & 107 \\
\hline 1833 & -- & 1872 & 124 & 1911 & 122 & 1950 & 116 & 1989 & 111 \\
\hline 1834 & -- & 1873 & 126 & 1912 & 117 & 1951 & 110 & 1990 & 106 \\
\hline 1835 & -- & 1874 & 129 & 1913 & 107 & 1952 & 111 & 1991 & 98 \\
\hline 1836 & -- & 1875 & 132 & 1914 & 121 & 1953 & 99 & 1992 & 114 \\
\hline 1837 & -- & 1876 & 132 & 1915 & 110 & 1954 & 106 & 1993 & 112 \\
\hline 1838 & -- & 1877 & 115 & 1916 & 122 & 1955 & 112 & 1994 & 110 \\
\hline 1839 & -- & 1878 & 108 & 1917 & 127 & 1956 & 127 & 1995 & 104 \\
\hline 1840 & -- & 1879 & 132 & 1918 & 116 & 1957 & 110 & 1996 & 117 \\
\hline 1841 & -- & 1880 & 111 & 1919 & 104 & 1958 & 114 & 1997 & 114 \\
\hline 1842 & -- & 1881 & 126 & 1920 & 120 & 1959 & 116 & 1998 & 103 \\
\hline 1843 & -- & 1882 & 119 & 1921 & 88 & 1960 & 115 & 1999 & 103 \\
\hline 1844 & -- & 1883 & 127 & 1922 & 96 & 1961 & 123 & 2000 & 100 \\
\hline 1845 & -- & 1884 & 123 & 1923 & 117 & 1962 & 116 & & \\
\hline
\end{tabular}


Table 25. Ice-out dates for Swan Lake, Maine

[--, no data]

Location of Lake.-Lat. $44^{\circ} 32^{\prime} 47^{\prime \prime}$, long. 68 59'38".

Period of Record.-1891 to 2000.

Years of Record. -110 .

Observer.-The Republican Journal.

Earliest Ice-Out Date.-1953: 86.

Latest Ice-Out Date.-1939: 127.

\begin{tabular}{|c|c|c|c|c|c|c|c|c|c|}
\hline Year & $\begin{array}{c}\text { Julian } \\
\text { day }\end{array}$ & Year & $\begin{array}{c}\text { Julian } \\
\text { day }\end{array}$ & Year & $\begin{array}{c}\text { Julian } \\
\text { day }\end{array}$ & Year & $\begin{array}{c}\text { Julian } \\
\text { day }\end{array}$ & Year & $\begin{array}{c}\text { Julian } \\
\text { day }\end{array}$ \\
\hline 1807 & -- & 1846 & -- & 1885 & -- & 1924 & 109 & 1963 & 112 \\
\hline 1808 & -- & 1847 & -- & 1886 & -- & 1925 & 94 & 1964 & 116 \\
\hline 1809 & -- & 1848 & -- & 1887 & -- & 1926 & 123 & 1965 & 111 \\
\hline 1810 & -- & 1849 & -- & 1888 & -- & 1927 & 107 & 1966 & 110 \\
\hline 1811 & -- & 1850 & -- & 1889 & -- & 1928 & 112 & 1967 & 121 \\
\hline 1812 & -- & 1851 & -- & 1890 & -- & 1929 & 111 & 1968 & 107 \\
\hline 1813 & -- & 1852 & -- & 1891 & 109 & 1930 & 104 & 1969 & 115 \\
\hline 1814 & -- & 1853 & -- & 1892 & 114 & 1931 & 103 & 1970 & 115 \\
\hline 1815 & -- & 1854 & -- & 1893 & 124 & 1932 & 108 & 1971 & 121 \\
\hline 1816 & -- & 1855 & -- & 1894 & 113 & 1933 & 108 & 1972 & 122 \\
\hline 1817 & -- & 1856 & -- & 1895 & 112 & 1934 & 114 & 1973 & 106 \\
\hline 1818 & -- & 1857 & -- & 1896 & 110 & 1935 & 116 & 1974 & 100 \\
\hline 1819 & -- & 1858 & -- & 1897 & 115 & 1936 & 98 & 1975 & 116 \\
\hline 1820 & -- & 1859 & -- & 1898 & 109 & 1937 & 106 & 1976 & 103 \\
\hline 1821 & -- & 1860 & -- & 1899 & 117 & 1938 & 106 & 1977 & 109 \\
\hline 1822 & -- & 1861 & -- & 1900 & 109 & 1939 & 127 & 1978 & 116 \\
\hline 1823 & -- & 1862 & -- & 1901 & 112 & 1940 & 122 & 1979 & 95 \\
\hline 1824 & -- & 1863 & -- & 1902 & 90 & 1941 & 108 & 1980 & 103 \\
\hline 1825 & -- & 1864 & -- & 1903 & 92 & 1942 & 112 & 1981 & 88 \\
\hline 1826 & -- & 1865 & -- & 1904 & 122 & 1943 & 114 & 1982 & 107 \\
\hline 1827 & -- & 1866 & -- & 1905 & 110 & 1944 & 119 & 1983 & 97 \\
\hline 1828 & -- & 1867 & -- & 1906 & 114 & 1945 & 93 & 1984 & 110 \\
\hline 1829 & -- & 1868 & -- & 1907 & 120 & 1946 & 101 & 1985 & 97 \\
\hline 1830 & -- & 1869 & -- & 1908 & 115 & 1947 & 102 & 1986 & 99 \\
\hline 1831 & -- & 1870 & -- & 1909 & 108 & 1948 & 102 & 1987 & 106 \\
\hline 1832 & -- & 1871 & -- & 1910 & 92 & 1949 & 99 & 1988 & 100 \\
\hline 1833 & -- & 1872 & -- & 1911 & 120 & 1950 & 111 & 1989 & 110 \\
\hline 1834 & -- & 1873 & -- & 1912 & 115 & 1951 & 96 & 1990 & 101 \\
\hline 1835 & -- & 1874 & -- & 1913 & 99 & 1952 & 108 & 1991 & 99 \\
\hline 1836 & -- & 1875 & -- & 1914 & 119 & 1953 & 86 & 1992 & 111 \\
\hline 1837 & -- & 1876 & -- & 1915 & 101 & 1954 & 110 & 1993 & 114 \\
\hline 1838 & -- & 1877 & -- & 1916 & 113 & 1955 & 106 & 1994 & 108 \\
\hline 1839 & -- & 1878 & -- & 1917 & 118 & 1956 & 120 & 1995 & 104 \\
\hline 1840 & -- & 1879 & -- & 1918 & 116 & 1957 & 104 & 1996 & 105 \\
\hline 1841 & -- & 1880 & -- & 1919 & 98 & 1958 & 109 & 1997 & 113 \\
\hline 1842 & -- & 1881 & -- & 1920 & 117 & 1959 & 112 & 1998 & 94 \\
\hline 1843 & -- & 1882 & -- & 1921 & 88 & 1960 & 112 & 1999 & 89 \\
\hline 1844 & -- & 1883 & -- & 1922 & 104 & 1961 & 123 & 2000 & 97 \\
\hline 1845 & -- & 1884 & -- & 1923 & 119 & 1962 & 112 & & \\
\hline
\end{tabular}


Table 26. Ice-out dates for Thompson Lake, Maine

[--, no data $]$

Location of Lake.-Lat. $44^{\circ} 03^{\prime} 58^{\prime \prime}$, long. $70^{\circ} 29^{\prime} 07^{\prime \prime}$.

Period of Record.-1902 to 2000.

Years of Record.- 99.

Observers.- Joseph M. Trebilcook, Richard Greenleaf, Thompson Lake Environmental Association.

Earliest Ice-Out Date.-1981: 88.

Latest Ice-Out Date.-1940: 128.

\begin{tabular}{|c|c|c|c|c|c|c|c|c|c|}
\hline Year & $\begin{array}{c}\text { Julian } \\
\text { day }\end{array}$ & Year & $\begin{array}{c}\text { Julian } \\
\text { day }\end{array}$ & Year & $\begin{array}{c}\text { Julian } \\
\text { day }\end{array}$ & Year & $\begin{array}{c}\text { Julian } \\
\text { day }\end{array}$ & Year & $\begin{array}{c}\text { Julian } \\
\text { day }\end{array}$ \\
\hline 1807 & -- & 1846 & -- & 1885 & -- & 1924 & 110 & 1963 & 110 \\
\hline 1808 & -- & 1847 & -- & 1886 & -- & 1925 & 100 & 1964 & 118 \\
\hline 1809 & -- & 1848 & -- & 1887 & -- & 1926 & 123 & 1965 & 119 \\
\hline 1810 & -- & 1849 & -- & 1888 & -- & 1927 & 107 & 1966 & 112 \\
\hline 1811 & -- & 1850 & -- & 1889 & -- & 1928 & 112 & 1967 & 118 \\
\hline 1812 & -- & 1851 & -- & 1890 & -- & 1929 & 113 & 1968 & 107 \\
\hline 1813 & -- & 1852 & -- & 1891 & -- & 1930 & 114 & 1969 & 110 \\
\hline 1814 & -- & 1853 & -- & 1892 & -- & 1931 & 102 & 1970 & 118 \\
\hline 1815 & -- & 1854 & -- & 1893 & -- & 1932 & 112 & 1971 & 123 \\
\hline 1816 & -- & 1855 & -- & 1894 & -- & 1933 & 114 & 1972 & 127 \\
\hline 1817 & -- & 1856 & -- & 1895 & -- & 1934 & 113 & 1973 & 110 \\
\hline 1818 & -- & 1857 & -- & 1896 & -- & 1935 & 113 & 1974 & 106 \\
\hline 1819 & -- & 1858 & -- & 1897 & -- & 1936 & 104 & 1975 & 103 \\
\hline 1820 & -- & 1859 & -- & 1898 & -- & 1937 & 117 & 1976 & 107 \\
\hline 1821 & -- & 1860 & -- & 1899 & -- & 1938 & 107 & 1977 & 109 \\
\hline 1822 & -- & 1861 & -- & 1900 & -- & 1939 & 126 & 1978 & 121 \\
\hline 1823 & -- & 1862 & -- & 1901 & -- & 1940 & 128 & 1979 & 104 \\
\hline 1824 & -- & 1863 & -- & 1902 & 107 & 1941 & 105 & 1980 & 103 \\
\hline 1825 & -- & 1864 & -- & 1903 & 94 & 1942 & 108 & 1981 & 88 \\
\hline 1826 & -- & 1865 & -- & 1904 & 121 & 1943 & 116 & 1982 & 118 \\
\hline 1827 & -- & 1866 & -- & 1905 & 113 & 1944 & 120 & 1983 & 100 \\
\hline 1828 & -- & 1867 & -- & 1906 & 113 & 1945 & 93 & 1984 & 112 \\
\hline 1829 & -- & 1868 & -- & 1907 & 120 & 1946 & 96 & 1985 & 101 \\
\hline 1830 & -- & 1869 & -- & 1908 & 116 & 1947 & 106 & 1986 & 102 \\
\hline 1831 & -- & 1870 & -- & 1909 & 110 & 1948 & 100 & 1987 & 100 \\
\hline 1832 & -- & 1871 & -- & 1910 & 95 & 1949 & 100 & 1988 & 102 \\
\hline 1833 & -- & 1872 & -- & 1911 & 118 & 1950 & 114 & 1989 & 118 \\
\hline 1834 & -- & 1873 & -- & 1912 & 94 & 1951 & 100 & 1990 & 105 \\
\hline 1835 & -- & 1874 & -- & 1913 & 108 & 1952 & 111 & 1991 & 99 \\
\hline 1836 & -- & 1875 & -- & 1914 & 113 & 1953 & 96 & 1992 & 101 \\
\hline 1837 & -- & 1876 & -- & 1915 & 105 & 1954 & 110 & 1993 & 113 \\
\hline 1838 & -- & 1877 & -- & 1916 & 110 & 1955 & 107 & 1994 & 114 \\
\hline 1839 & -- & 1878 & -- & 1917 & 118 & 1956 & 123 & 1995 & 104 \\
\hline 1840 & -- & 1879 & -- & 1918 & 114 & 1957 & 107 & 1996 & 106 \\
\hline 1841 & -- & 1880 & -- & 1919 & 102 & 1958 & 109 & 1997 & 113 \\
\hline 1842 & -- & 1881 & -- & 1920 & 116 & 1959 & 113 & 1998 & 99 \\
\hline 1843 & -- & 1882 & -- & 1921 & 93 & 1960 & 116 & 1999 & 96 \\
\hline 1844 & -- & 1883 & -- & 1922 & 107 & 1961 & 121 & 2000 & 99 \\
\hline 1845 & -- & 1884 & -- & 1923 & 114 & 1962 & 113 & & \\
\hline
\end{tabular}


Table 27. Ice-out dates for Umbagog Lake, New Hampshire/Maine

[--, no data]

Location of Lake.-Lat. $44^{\circ} 48^{\prime} 05^{\prime \prime}$, long. $71^{\circ} 01^{\prime} 42^{\prime \prime}$.

Period of Record.-1880 to 2000.

Years of Record. -110 .

Observers. - Union Water Power Company, FPL Energy Maine.

Earliest Ice-Out Date.-1910, 1921: 100.

Latest Ice-Out Date.-1888: 144.

\begin{tabular}{|c|c|c|c|c|c|c|c|c|c|}
\hline Year & $\begin{array}{c}\text { Julian } \\
\text { day }\end{array}$ & Year & $\begin{array}{c}\text { Julian } \\
\text { day }\end{array}$ & Year & $\begin{array}{c}\text { Julian } \\
\text { day }\end{array}$ & Year & $\begin{array}{c}\text { Julian } \\
\text { day }\end{array}$ & Year & $\begin{array}{c}\text { Julian } \\
\text { day }\end{array}$ \\
\hline 1807 & -- & 1846 & -- & 1885 & -- & 1924 & 124 & 1963 & 119 \\
\hline 1808 & -- & 1847 & -- & 1886 & -- & 1925 & 116 & 1964 & 123 \\
\hline 1809 & -- & 1848 & -- & 1887 & -- & 1926 & 129 & 1965 & 124 \\
\hline 1810 & -- & 1849 & -- & 1888 & 144 & 1927 & 110 & 1966 & 123 \\
\hline 1811 & -- & 1850 & -- & 1889 & 119 & 1928 & 130 & 1967 & 122 \\
\hline 1812 & -- & 1851 & -- & 1890 & 131 & 1929 & 121 & 1968 & 110 \\
\hline 1813 & -- & 1852 & -- & 1891 & -- & 1930 & 122 & 1969 & 126 \\
\hline 1814 & -- & 1853 & -- & 1892 & -- & 1931 & 111 & 1970 & 130 \\
\hline 1815 & -- & 1854 & -- & 1893 & 141 & 1932 & 121 & 1971 & 129 \\
\hline 1816 & -- & 1855 & -- & 1894 & -- & 1933 & 125 & 1972 & 135 \\
\hline 1817 & -- & 1856 & -- & 1895 & -- & 1934 & 116 & 1973 & 117 \\
\hline 1818 & -- & 1857 & -- & 1896 & 123 & 1935 & 125 & 1974 & 130 \\
\hline 1819 & -- & 1858 & -- & 1897 & -- & 1936 & 121 & 1975 & 129 \\
\hline 1820 & -- & 1859 & -- & 1898 & -- & 1937 & 129 & 1976 & 113 \\
\hline 1821 & -- & 1860 & -- & 1899 & 128 & 1938 & 117 & 1977 & 118 \\
\hline 1822 & -- & 1861 & -- & 1900 & -- & 1939 & 131 & 1978 & 132 \\
\hline 1823 & -- & 1862 & -- & 1901 & 119 & 1940 & 131 & 1979 & 117 \\
\hline 1824 & -- & 1863 & -- & 1902 & 112 & 1941 & 109 & 1980 & 117 \\
\hline 1825 & -- & 1864 & -- & 1903 & 106 & 1942 & 121 & 1981 & 111 \\
\hline 1826 & -- & 1865 & -- & 1904 & 114 & 1943 & 131 & 1982 & 127 \\
\hline 1827 & -- & 1866 & -- & 1905 & 119 & 1944 & 126 & 1983 & 122 \\
\hline 1828 & -- & 1867 & -- & 1906 & 131 & 1945 & 103 & 1984 & 121 \\
\hline 1829 & -- & 1868 & -- & 1907 & 131 & 1946 & 116 & 1985 & 121 \\
\hline 1830 & -- & 1869 & -- & 1908 & 122 & 1947 & 128 & 1986 & 110 \\
\hline 1831 & -- & 1870 & -- & 1909 & 134 & 1948 & 115 & 1987 & 108 \\
\hline 1832 & -- & 1871 & -- & 1910 & 100 & 1949 & 112 & 1988 & 115 \\
\hline 1833 & -- & 1872 & -- & 1911 & 126 & 1950 & 126 & 1989 & 126 \\
\hline 1834 & -- & 1873 & -- & 1912 & 124 & 1951 & 118 & 1990 & 118 \\
\hline 1835 & -- & 1874 & -- & 1913 & 120 & 1952 & 118 & 1991 & 118 \\
\hline 1836 & -- & 1875 & -- & 1914 & 130 & 1953 & 114 & 1992 & 130 \\
\hline 1837 & -- & 1876 & -- & 1915 & 116 & 1954 & 122 & 1993 & 121 \\
\hline 1838 & -- & 1877 & -- & 1916 & 125 & 1955 & 114 & 1994 & 125 \\
\hline 1839 & -- & 1878 & -- & 1917 & 130 & 1956 & 128 & 1995 & 116 \\
\hline 1840 & -- & 1879 & -- & 1918 & 118 & 1957 & 115 & 1996 & 125 \\
\hline 1841 & -- & 1880 & 129 & 1919 & 121 & 1958 & 122 & 1997 & 123 \\
\hline 1842 & -- & 1881 & 135 & 1920 & 129 & 1959 & 117 & 1998 & 109 \\
\hline 1843 & -- & 1882 & 141 & 1921 & 100 & 1960 & 119 & 1999 & 119 \\
\hline 1844 & -- & 1883 & 134 & 1922 & 117 & 1961 & 130 & 2000 & 113 \\
\hline 1845 & -- & 1884 & -- & 1923 & 123 & 1962 & 122 & & \\
\hline
\end{tabular}


Table 28. Ice-out dates for West Grand Lake, Maine

[--, no data]

Location of Lake.-Lat. $45^{\circ} 12^{\prime} 56^{\prime \prime}$, long. $67^{\circ} 48^{\prime} 52^{\prime \prime}$.

Period of Record.-1878 to 2000.

Years of Record.-123.

Observers.-Marion Staples and family (three generations).

Earliest Ice-Out Date.—1999: 97.

Latest Ice-Out Date._-1918: 139.

\begin{tabular}{|c|c|c|c|c|c|c|c|c|c|}
\hline Year & $\begin{array}{c}\text { Julian } \\
\text { day }\end{array}$ & Year & $\begin{array}{c}\text { Julian } \\
\text { day }\end{array}$ & Year & $\begin{array}{c}\text { Julian } \\
\text { day }\end{array}$ & Year & $\begin{array}{c}\text { Julian } \\
\text { day }\end{array}$ & Year & $\begin{array}{c}\text { Julian } \\
\text { day }\end{array}$ \\
\hline 1807 & -- & 1846 & -- & 1885 & 125 & 1924 & 128 & 1963 & 124 \\
\hline 1808 & -- & 1847 & -- & 1886 & 123 & 1925 & 104 & 1964 & 124 \\
\hline 1809 & -- & 1848 & -- & 1887 & 131 & 1926 & 134 & 1965 & 122 \\
\hline 1810 & -- & 1849 & -- & 1888 & 138 & 1927 & 113 & 1966 & 115 \\
\hline 1811 & -- & 1850 & -- & 1889 & 116 & 1928 & 124 & 1967 & 125 \\
\hline 1812 & -- & 1851 & -- & 1890 & 128 & 1929 & 120 & 1968 & 112 \\
\hline 1813 & -- & 1852 & -- & 1891 & 124 & 1930 & 117 & 1969 & 124 \\
\hline 1814 & -- & 1853 & -- & 1892 & 121 & 1931 & 111 & 1970 & 121 \\
\hline 1815 & -- & 1854 & -- & 1893 & 129 & 1932 & 116 & 1971 & 123 \\
\hline 1816 & -- & 1855 & -- & 1894 & 120 & 1933 & 119 & 1972 & 135 \\
\hline 1817 & -- & 1856 & -- & 1895 & 122 & 1934 & 115 & 1973 & 117 \\
\hline 1818 & -- & 1857 & -- & 1896 & 120 & 1935 & 125 & 1974 & 112 \\
\hline 1819 & -- & 1858 & -- & 1897 & 124 & 1936 & 107 & 1975 & 124 \\
\hline 1820 & -- & 1859 & -- & 1898 & 117 & 1937 & 119 & 1976 & 111 \\
\hline 1821 & -- & 1860 & -- & 1899 & 117 & 1938 & 115 & 1977 & 122 \\
\hline 1822 & -- & 1861 & -- & 1900 & 119 & 1939 & 131 & 1978 & 127 \\
\hline 1823 & -- & 1862 & -- & 1901 & 115 & 1940 & 129 & 1979 & 113 \\
\hline 1824 & -- & 1863 & -- & 1902 & 98 & 1941 & 117 & 1980 & 109 \\
\hline 1825 & -- & 1864 & -- & 1903 & 110 & 1942 & 120 & 1981 & 100 \\
\hline 1826 & -- & 1865 & -- & 1904 & 126 & 1943 & 121 & 1982 & 124 \\
\hline 1827 & -- & 1866 & -- & 1905 & 118 & 1944 & 123 & 1983 & 104 \\
\hline 1828 & -- & 1867 & -- & 1906 & 125 & 1945 & 103 & 1984 & 121 \\
\hline 1829 & -- & 1868 & -- & 1907 & 125 & 1946 & 117 & 1985 & 113 \\
\hline 1830 & -- & 1869 & -- & 1908 & 121 & 1947 & 114 & 1986 & 113 \\
\hline 1831 & -- & 1870 & -- & 1909 & 126 & 1948 & 116 & 1987 & 112 \\
\hline 1832 & -- & 1871 & -- & 1910 & 100 & 1949 & 106 & 1988 & 111 \\
\hline 1833 & -- & 1872 & -- & 1911 & 123 & 1950 & 120 & 1989 & 126 \\
\hline 1834 & -- & 1873 & -- & 1912 & 128 & 1951 & 103 & 1990 & 117 \\
\hline 1835 & -- & 1874 & -- & 1913 & 110 & 1952 & 120 & 1991 & 118 \\
\hline 1836 & -- & 1875 & -- & 1914 & 130 & 1953 & 98 & 1992 & 124 \\
\hline 1837 & -- & 1876 & -- & 1915 & 110 & 1954 & 118 & 1993 & 117 \\
\hline 1838 & -- & 1877 & -- & 1916 & 118 & 1955 & 115 & 1994 & 122 \\
\hline 1839 & -- & 1878 & 116 & 1917 & 124 & 1956 & 126 & 1995 & 118 \\
\hline 1840 & -- & 1879 & 130 & 1918 & 139 & 1957 & 119 & 1996 & 115 \\
\hline 1841 & -- & 1880 & 123 & 1919 & 119 & 1958 & 115 & 1997 & 118 \\
\hline 1842 & -- & 1881 & 123 & 1920 & 125 & 1959 & 124 & 1998 & 107 \\
\hline 1843 & -- & 1882 & 126 & 1921 & 100 & 1960 & 122 & 1999 & 97 \\
\hline 1844 & -- & 1883 & 129 & 1922 & 108 & 1961 & 132 & 2000 & 100 \\
\hline 1845 & -- & 1884 & 121 & 1923 & 126 & 1962 & 119 & & \\
\hline
\end{tabular}


Table 29. Ice-out dates for Lake Winnipesaukee, New Hampshire

[--, no data]

Location of Lake.-Lat. $43^{\circ} 35^{\prime} 56^{\prime \prime}$, long. $71^{\circ} 19^{\prime} 21^{\prime \prime}$.

Period of Record.-1887 to 2000.

Years of Record. - 114 .

Observers and Ice-Out Definition.-New Hampshire Office of Vacation Travel, Harry Daniel, Arthur Marshall, Bob Aldrich, The Lakes Region Association, The Classic Journey Company. Ice-out definition for most years thought be date of open navigation for the Mount Washington on its regular route, except for Meredith Bay.

Earliest Ice-Out Date.-1921: 87.

Latest Ice-Out Date.-1888: 133.

\begin{tabular}{|c|c|c|c|c|c|c|c|c|c|}
\hline Year & $\begin{array}{c}\text { Julian } \\
\text { day }\end{array}$ & Year & $\begin{array}{c}\text { Julian } \\
\text { day }\end{array}$ & Year & $\begin{array}{c}\text { Julian } \\
\text { day }\end{array}$ & Year & $\begin{array}{c}\text { Julian } \\
\text { day }\end{array}$ & Year & $\begin{array}{c}\text { Julian } \\
\text { day }\end{array}$ \\
\hline 1807 & -- & 1846 & -- & 1885 & -- & 1924 & 109 & 1963 & 110 \\
\hline 1808 & -- & 1847 & -- & 1886 & -- & 1925 & 100 & 1964 & 119 \\
\hline 1809 & -- & 1848 & -- & 1887 & 127 & 1926 & 122 & 1965 & 112 \\
\hline 1810 & -- & 1849 & -- & 1888 & 133 & 1927 & 103 & 1966 & 110 \\
\hline 1811 & -- & 1850 & -- & 1889 & 104 & 1928 & 110 & 1967 & 110 \\
\hline 1812 & -- & 1851 & -- & 1890 & 114 & 1929 & 108 & 1968 & 106 \\
\hline 1813 & -- & 1852 & -- & 1891 & 113 & 1930 & 97 & 1969 & 115 \\
\hline 1814 & -- & 1853 & -- & 1892 & 102 & 1931 & 101 & 1970 & 118 \\
\hline 1815 & -- & 1854 & -- & 1893 & 130 & 1932 & 111 & 1971 & 126 \\
\hline 1816 & -- & 1855 & -- & 1894 & 110 & 1933 & 115 & 1972 & 123 \\
\hline 1817 & -- & 1856 & -- & 1895 & 116 & 1934 & 111 & 1973 & 106 \\
\hline 1818 & -- & 1857 & -- & 1896 & 114 & 1935 & 111 & 1974 & 106 \\
\hline 1819 & -- & 1858 & -- & 1897 & 113 & 1936 & 99 & 1975 & 115 \\
\hline 1820 & -- & 1859 & -- & 1898 & 104 & 1937 & 115 & 1976 & 107 \\
\hline 1821 & -- & 1860 & -- & 1899 & 122 & 1938 & 107 & 1977 & 113 \\
\hline 1822 & -- & 1861 & -- & 1900 & 116 & 1939 & 124 & 1978 & 117 \\
\hline 1823 & -- & 1862 & -- & 1901 & 110 & 1940 & 125 & 1979 & 116 \\
\hline 1824 & -- & 1863 & -- & 1902 & 94 & 1941 & 106 & 1980 & 108 \\
\hline 1825 & -- & 1864 & -- & 1903 & 92 & 1942 & 108 & 1981 & 95 \\
\hline 1826 & -- & 1865 & -- & 1904 & 120 & 1943 & 120 & 1982 & 119 \\
\hline 1827 & -- & 1866 & -- & 1905 & 114 & 1944 & 124 & 1983 & 100 \\
\hline 1828 & -- & 1867 & -- & 1906 & 116 & 1945 & 91 & 1984 & 111 \\
\hline 1829 & -- & 1868 & -- & 1907 & 119 & 1946 & 89 & 1985 & 104 \\
\hline 1830 & -- & 1869 & -- & 1908 & 112 & 1947 & 114 & 1986 & 106 \\
\hline 1831 & -- & 1870 & -- & 1909 & 109 & 1948 & 101 & 1987 & 102 \\
\hline 1832 & -- & 1871 & -- & 1910 & 96 & 1949 & 108 & 1988 & 107 \\
\hline 1833 & -- & 1872 & -- & 1911 & 122 & 1950 & 110 & 1989 & 115 \\
\hline 1834 & -- & 1873 & -- & 1912 & 114 & 1951 & 104 & 1990 & 112 \\
\hline 1835 & -- & 1874 & -- & 1913 & 107 & 1952 & 111 & 1991 & 98 \\
\hline 1836 & -- & 1875 & -- & 1914 & 105 & 1953 & 93 & 1992 & 112 \\
\hline 1837 & -- & 1876 & -- & 1915 & 114 & 1954 & 106 & 1993 & 112 \\
\hline 1838 & -- & 1877 & -- & 1916 & 107 & 1955 & 109 & 1994 & 113 \\
\hline 1839 & -- & 1878 & -- & 1917 & 118 & 1956 & 126 & 1995 & 105 \\
\hline 1840 & -- & 1879 & -- & 1918 & 114 & 1957 & 93 & 1996 & 108 \\
\hline 1841 & -- & 1880 & -- & 1919 & 104 & 1958 & 103 & 1997 & 114 \\
\hline 1842 & -- & 1881 & -- & 1920 & 115 & 1959 & 116 & 1998 & 97 \\
\hline 1843 & -- & 1882 & -- & 1921 & 87 & 1960 & 110 & 1999 & 98 \\
\hline 1844 & -- & 1883 & -- & 1922 & 107 & 1961 & 117 & 2000 & 101 \\
\hline 1845 & -- & 1884 & -- & 1923 & 114 & 1962 & 114 & & \\
\hline
\end{tabular}




District Chief

Maine District

U.S. Geological Survey

Water Resources Division

26 Ganneston Drive

Augusta, Maine 04330 